\title{
Recent understanding of the oxidation and burning of magnesium alloys
}

Qiyang Tan PhD

Postdoctoral Research Fellow, School of Mechanical and Mining Engineering, University of Queensland, St Lucia, Australia

Yu Yin MSc

PhD candidate, School of Mechanical and Mining Engineering, University of Queensland, St Lucia, Australia

Ning Mo BSC

$\mathrm{PhD}$ candidate, School of Mechanical and Mining Engineering,

University of Queensland, St Lucia, Australia
Mingxing Zhang PhD

Professor, School of Mechanical and Mining Engineering, University of Queensland, St Lucia, Australia

Andrej Atrens PhD

Professor, School of Mechanical and Mining Engineering, University of Queensland, St Lucia, Australia (corresponding author: andrejs.atrens@uq.edu.au)

This paper reviews the following for magnesium (Mg) alloys: (a) fundamentals of oxidation and burning; (b) influence of alloying on oxidation; (c) theoretical models for improved oxidation resistance through alloying with calcium (Ca), beryllium (Be), strontium ( $\mathrm{Sr}$ ) and rare earth elements; $(d)$ recent research on the improved oxidation resistance of beryllium-containing magnesium alloys; and (e) the recent proposed oxide reinforcement model. The oxidation resistance of magnesium alloys is closely related to the protective incubation period, which is mainly controlled by the crack resistance of the initially formed surface oxide layer. Cracking of the initially formed surface oxide facilitates the vapor phase diffusion of magnesium and results in catastrophic oxidation and eventual burning of magnesium. According to the oxide reinforcement model, an oxide layer with increased mechanical strength and hardness (such as the ( $\mathrm{Mg}, \mathrm{Be}) \mathrm{O}$ layer on beryllium-containing alloys) can better withstand the internal tensile stress arising during the oxidation process and therefore can delay oxide layer cracking and extend the oxidation incubation period. This model provides guidance on developing new oxidation-resistant magnesium alloys.

\section{Notation}

a lattice parameter

c mole atomic concentration

d layer thickness

$d \quad$ interplanar distance of a crystallographic plane $(\AA)$

$H \quad$ hardness

$H_{1} \quad$ hardness of the oxide layer

$H_{\mathrm{s}} \quad$ hardness of the substrate

$h \quad$ indenter penetration depth

$h k l \quad$ Miller indices of a crystallographic plane

$M \quad$ atomic or molecular mass

$n$

$p$

$R$

$R_{\mathrm{a}}$

$R_{\mathrm{PB}}$

$r_{\mathrm{S}}$

$T$

$T_{\mathrm{i}}$

$V_{\mathrm{m}}$

$V_{\text {ox }} \quad$ volume of the elementary cell of the metal oxide

$X \quad$ element

$X_{\mathrm{Re}} \quad$ reactive element

$X_{\mathrm{Sa}} \quad$ surface-active element

$\alpha \quad$ activity

$\Delta G^{0} \quad$ standard Gibbs free energy of formation

$\rho$ density

\section{Introduction}

The urgent demand for lighter materials in the automobile, railway and aerospace industries to enable higher fuel efficiency and less greenhouse gas emissions has been driven by the current shortages in energy and fuel and increasing carbon dioxide $\left(\mathrm{CO}_{2}\right)$ emissions. As the lightest structural metallic material, magnesium $(\mathrm{Mg})$ alloys are gaining increasing interest because of their high specific strength, high stiffness, good castability and good damping capacity. ${ }^{1-5}$ Consequently, the global production of primary magnesium has increased each year in the past decade and reached $1.1 \mathrm{Mt}$ in $2017 .^{6}$ At present, the application of magnesium alloys has included components in vehicles such as steering wheels, instrument panels, seats and gearboxes and parts in aircraft such as the thrust reverser cascade, wheels, drive train components and transmission casings. ${ }^{7-10}$ However, the widespread application of magnesium alloys is restricted because of their limited cold workability, poor creep resistance and low wear resistance, ${ }^{7,11}$ particularly compared with steels and aluminium (Al) alloys.

Furthermore, magnesium has high chemical reactivity, which causes susceptibility to aqueous corrosion and oxidation in air at elevated temperatures. ${ }^{12-19}$ The magnesium oxide $(\mathrm{MgO})$ layer initially formed on magnesium alloys during air oxidation has an incompact structure with a low Pilling-Bedworth $(\mathrm{P}-\mathrm{B})$ ratio $\left(R_{\mathrm{PB}}\right)$ of $0 \cdot 81,{ }^{13,20,21}$ unlike the protective oxide layers on aluminium, stainless steel or titanium (Ti) alloys. Thickening of the magnesium 
oxide layer during oxidation in air at high temperatures is accompanied by an increase in the internal tension stress, leading to layer cracking and subsequent rapid oxidation. ${ }^{13}$

The major problems caused by the low oxidation resistance of magnesium alloys are (a) surface degradation during long-term exposure at elevated temperatures and $(b)$ easy burning when in contact with a sufficiently strong heat or flame, particularly when the magnesium alloy is in the form of fine powder or fragmented chips, although resistance is much higher for larger sections. ${ }^{12,13,22}$ Consequently, magnesium alloys have long been restricted from use in aircraft interiors, in particular the passenger cabin, due to concerns with magnesium burning, ${ }^{23,24}$ in contrast to other lightweight metals, such as titanium- and aluminium-based alloys. A recent full-scale simulated test conducted by the Federal Aviation Administration (FAA) indicated that most commercial magnesium alloys, such as AZ31, AZ80 and ZE41, were unable to meet the survivability requirement in the passenger cabin, whereas some rare-earth (RE)-containing magnesium alloys were significantly better than existing aluminium alloys. ${ }^{25}$ Hence, it is of critical significance to develop magnesium alloys with improved oxidation resistance for their wider applications.

The present paper reviews $(a)$ the fundamentals of the oxidation of magnesium alloys, $(b)$ the current models of the effect of alloying elements on the oxidation resistance of magnesium alloys, $(c)$ recent research on the oxidation behaviour of beryllium (Be)-containing magnesium alloys and (d) the new oxide reinforcement $(\mathrm{OR})$ model that provides a new theoretical basis for the development of new oxidation-resistant magnesium alloys.

\section{Oxidation}

\section{$2.1 \quad$ Oxidation kinetics}

Magnesium alloys are easily oxidised when exposed to air at high temperatures, due to a high affinity to oxygen $\left(\mathrm{O}_{2}\right){ }^{26}$ The chemical reaction between magnesium and oxygen is as follows ${ }^{27}$

$$
\text { 1. } \mathrm{Mg}+\frac{1}{2} \mathrm{O}_{2}=\mathrm{MgO}
$$

As oxidation progresses, the oxide layer thickens and the weight increases. ${ }^{28}$ The weight gain of the magnesium alloy specimen is equivalent to the mass of oxygen taken into the oxide layer according to Equation 1. Hence, the oxidation kinetics can be characterised by measuring the weight gain of the magnesium alloy specimen, because it has been experimentally found that the oxide layer remains on the alloy surface.

Figure 1 presents typical weight gain-time curves, as measured by thermogravimetric analysis (TGA), for AZ91 in air at different temperatures. ${ }^{29}$ At $197^{\circ} \mathrm{C}$, the overall specific weight gain was as low as $\sim 0.02 \mathrm{mg} / \mathrm{cm}^{2}$, indicating slow oxidation. Higher temperatures above $400^{\circ} \mathrm{C}$ facilitated oxidation and caused a significantly increased weight gain. After oxidation at $487^{\circ} \mathrm{C}$

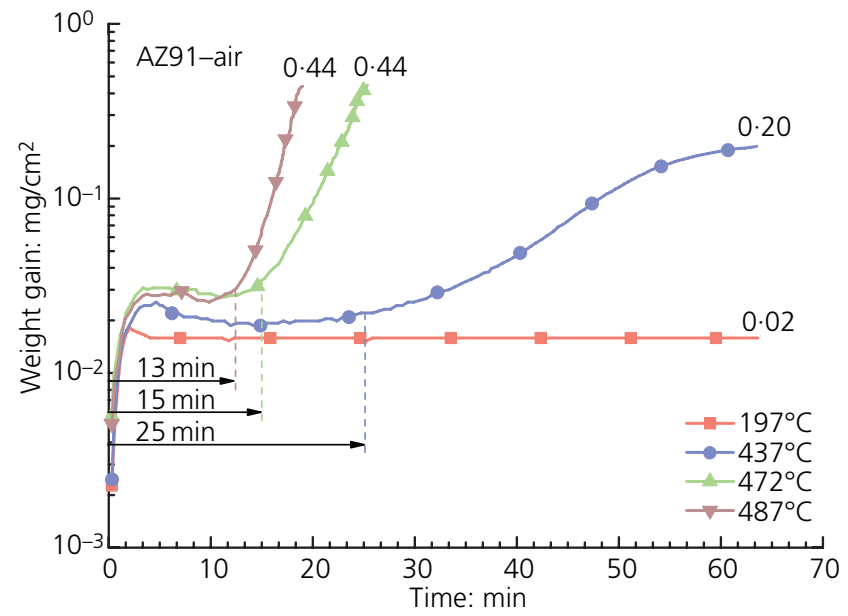

Figure 1. The weight gain-time curves for as-cast $\mathrm{Mg}-9 \mathrm{Al}-1 \mathrm{Zn}$ in air at $197,437,472$ and $487^{\circ} \mathrm{C}$ measured by TGA. At $197^{\circ} \mathrm{C}$, the overall weight gain was small, indicating a slow oxidation. Increasing the temperature to over $400^{\circ} \mathrm{C}$ led to accelerated oxidation stages after the incubation periods. Redrawn from Czerwinski ${ }^{29}$ and used with permission from Elsevier

for $\sim 19 \mathrm{~min}$, the specific overall weight gain of AZ91 was $0.44 \mathrm{mg} / \mathrm{cm}^{2}$, which was over 20 times higher than that at $197^{\circ} \mathrm{C}$.

High-temperature oxidation of magnesium alloys in air typically involves an initial oxidation stage with parabolic kinetics and a subsequent oxidation stage with accelerated kinetics which are linear or power-law kinetics. ${ }^{13,30}$ The parabolic growth stage is considered as an oxidation incubation period, during which a compact protective oxide layer forms and isolates the magnesium substrate from the oxygen in air. ${ }^{13,29}$ Within the incubation period, the oxidation is mainly dominated by ionic diffusion and the oxidation rate decreases with increasing layer thickness. ${ }^{28}$ In contrast, the accelerated oxidation stage represents non-protective oxidation, which is attributed to the breakage of the initially formed surface oxide layer. ${ }^{27,29,31}$ During the accelerated oxidation stage, the oxidation rate is independent of the oxide layer thickness, and oxidation proceeds with linear or power-law kinetics. ${ }^{28,32}$ Hence, the oxidation resistance of magnesium alloys is closely associated with the incubation period. As shown in Figure 1, the weight gain of the AZ91 followed parabolic kinetics within the entire test period at $197^{\circ} \mathrm{C}$, indicating that the surface oxide provided sufficient protection to the alloy at this relatively low temperature. In contrast, at a temperature higher than $400^{\circ} \mathrm{C}$, there was an accelerated oxidation stage after the incubation period. At $437^{\circ} \mathrm{C}, \mathrm{AZ} 91$ had an oxidation incubation period of $\sim 25 \mathrm{~min}$. Increasing the temperature to 472 and $487^{\circ} \mathrm{C}$ shortened the incubation period to $\sim 15$ and $\sim 13 \mathrm{~min}$, respectively. The rapid oxidation with linear kinetics at both 472 and $487^{\circ} \mathrm{C}$ indicated the occurrence of fast oxidation.

The oxidation kinetics of magnesium alloys thus has a critical temperature, which ranges from 400 to $450^{\circ} \mathrm{C}$ depending on the 
alloy composition. ${ }^{13}$ Below the critical temperature, there is a slow oxidation rate and a small weight gain. In contrast, above the critical temperature, the weight grain accelerates and follows linear or power-law kinetics after the incubation period. A higher oxidation temperature results in a higher oxidation rate and a shorter incubation period. ${ }^{30,33,34}$
The published data on incubation periods of magnesium alloys at various temperatures are summarised in Table $1 .^{13,21,30,33-55}$ Pure magnesium had an incubation period of $440 \mathrm{~min}$ at $500^{\circ} \mathrm{C}$. The alloying elements aluminium, zinc $(\mathrm{Zn})$, tin $(\mathrm{Sn})$, copper $(\mathrm{Cu})$ and manganese $(\mathrm{Mn})$ shorten the incubation period for magnesium alloys. Commercial magnesium alloys such as AZ31, AZ91, AM60, ZC63

Table 1. Summary of the incubation period of various magnesium alloys at various temperatures

\begin{tabular}{|c|c|c|c|c|c|c|c|c|}
\hline \multirow{2}{*}{ Alloy } & \multicolumn{7}{|c|}{ Incubation period at various temperatures: $\min$} & \multirow{2}{*}{ Reference } \\
\hline & $400^{\circ} \mathrm{C}$ & $430^{\circ} \mathrm{C}$ & $450^{\circ} \mathrm{C}$ & $500^{\circ} \mathrm{C}$ & $550^{\circ} \mathrm{C}$ & $600^{\circ} \mathrm{C}$ & $700^{\circ} \mathrm{C}$ & \\
\hline Pure magnesium & & & & 440 & & & & Aydin et $a l^{35}$ \\
\hline AZ31 & & & & 55 & & & & Yu et al. ${ }^{51}$ \\
\hline AZ61 & 350 & & & & & & & $\mathrm{Lee}^{52}$ \\
\hline \multirow[t]{3}{*}{ AZ91 } & 150 & & & & & & & Tan et al. ${ }^{45}$ \\
\hline & 180 & & 20 & & & & & Medved et al. ${ }^{33}$ \\
\hline & & & & 55 & & & & Barrena et al. ${ }^{54}$ \\
\hline AM50 & $>700$ & & 95 & 85 & & & & Medved et al. ${ }^{33}$ \\
\hline & & & 41 & & & & & Lin et al. ${ }^{48}$ \\
\hline AM60 & $>700$ & & $\begin{array}{l}80 \\
60\end{array}$ & 75 & & & & $\begin{array}{l}\text { Medved et al. } \\
\text { Aydin et al. }\end{array}$ \\
\hline AE42 & $>700$ & & 130 & 95 & & & & Medved et al. ${ }^{33}$ \\
\hline AM50-0.28Y & & & $>60$ & & & & & Lin et al. ${ }^{48}$ \\
\hline AM50-1Y & & & 57 & & & & & Lin et al. ${ }^{48}$ \\
\hline AM60-10SiC & & & $>300$ & & & & & Aydin et al. ${ }^{35}$ \\
\hline AS21 & & & & 140 & & & & Tan et al. 55 \\
\hline AZ31-0.3Ca & & & $>1200$ & $>1200$ & & & & $\operatorname{Lee}^{38}$ \\
\hline AZ31-0.75La & & & 36 & & & & & Zhao et al. ${ }^{44}$ \\
\hline$A Z 61-0 \cdot 5 C a$ & & & & $>1200$ & 60 & & & $\operatorname{Lee}^{52}$ \\
\hline AZ91-1.5Ca & 270 & & & & & & & Cheng et $a l^{37}$ \\
\hline AZ91-1.5Ca-0.5Y & $>300$ & & & & & & & Cheng et $a l^{37}$ \\
\hline AZ91-10SiC & & $>300$ & 90 & & & & & Nguyen et al. ${ }^{47}$ \\
\hline AZ91-20SiC & & $>300$ & $>300$ & & & & & Nguyen et al. ${ }^{47}$ \\
\hline AZ91-2Ca & & & & & & & 55 & Choi et al. ${ }^{49}$ \\
\hline AZ91-5Ca & & & & & & & $>60$ & Choi et al. ${ }^{49}$ \\
\hline AZ91-5SiC & & 120 & 60 & & & & & Nguyen et al. ${ }^{47}$ \\
\hline AZ91-60 ppm (wt) beryllium & $>300$ & & & & & & & Tan et al. ${ }^{45}$ \\
\hline $\mathrm{Mg}-0.3 \mathrm{Nd}$ & & & & 480 & & & & Aydin et al. ${ }^{35}$ \\
\hline $\mathrm{Mg}-0.5 \mathrm{Nd}$ & & & & 670 & & & & Aydin et al. ${ }^{35}$ \\
\hline $\mathrm{Mg}-0.5 \mathrm{Y}$ & & & & & $>100$ & 60 & & Yu et al. 21 \\
\hline $\mathrm{Mg}-1 \cdot 5 \mathrm{Ca}$ & & & & $>300$ & & & & You et al. ${ }^{36}$ \\
\hline $\mathrm{Mg}-1.5 \mathrm{Y}$ & & & & $>160$ & & & & Yu et al. ${ }^{51}$ \\
\hline $\mathrm{Mg}-1 \cdot 7 \mathrm{Ca}(\mathrm{OH})_{2}$ & & & & $>300$ & & & & Jang and $\mathrm{Kim}^{53}$ \\
\hline $\mathrm{Mg}-1 \cdot 7 \mathrm{CaO}$ & & & & $>300$ & & & & Jang and $\mathrm{Kim}^{53}$ \\
\hline$M g-10 G d-3 Y$ & $>90$ & & $>90$ & $>90$ & $>90$ & $>90$ & & Wang et al. ${ }^{42}$ \\
\hline $\mathrm{Mg}-1 \mathrm{Y}$ & & & & & $>100$ & 90 & & Yu et al. ${ }^{21}$ \\
\hline $\mathrm{Mg}-2 \cdot 5 \mathrm{Nd}$ & & & & $>700$ & & & & Aydin et al. ${ }^{43}$ \\
\hline $\mathrm{Mg}-2 \cdot 5 \mathrm{Sr}$ & & & & $>700$ & & & & Aydin et al. ${ }^{46}$ \\
\hline $\mathrm{Mg}-2 \mathrm{Sn}$ & & & & 60 & & & & Yu et al. ${ }^{51}$ \\
\hline$M g-2 Z n$ & & & & 30 & & & & Tan et al. ${ }^{55}$ \\
\hline $\mathrm{Mg}-3.08 \mathrm{Y}-0.68 \mathrm{Ce}$ & $>140$ & & & $>140$ & & 16 & & Fan et al. ${ }^{40}$ \\
\hline $\mathrm{Mg}-3 \cdot 7 \mathrm{Y}$ & & & & & $>100$ & $>100$ & & Yu et al..$^{21}$ \\
\hline$M g-5 \cdot 13 Y-0 \cdot 25 Z n-0 \cdot 32 Z r$ & $>16200$ & & & & & & & Ning et al. ${ }^{41}$ \\
\hline $\mathrm{Mg}-6 \mathrm{Nd}$ & & & & $>700$ & & & & Aydin et al. ${ }^{43}$ \\
\hline $\mathrm{Mg}-6 \mathrm{Sr}$ & & & & $>700$ & & & & Aydin et al. ${ }^{46}$ \\
\hline Yttrium-surface-implanted magnesium & & & & $>90$ & & & & Wang et al. ${ }^{39}$ \\
\hline ZC63 & & & & 15 & & & & Tan et al. ${ }^{55}$ \\
\hline ZC63-10SiC & & 90 & 55 & & & & & Lee et $a .^{34}$ \\
\hline ZE41 & $>330$ & & $>330$ & 90 & & & & López et al. ${ }^{30}$ \\
\hline ZK2O & & & & 20 & & & & Tan et al. ${ }^{55}$ \\
\hline
\end{tabular}

Chemical compositions are represented by mass fraction. The prefix ' $>$ ' indicates that the oxidation test was terminated within the incubation period. Reproduced from Tan et al..$^{13}$ and supplemented with updated data ${ }^{21,30,33-55}$ and used with permission from Elsevier 
and ZK20 exhibited incubation periods shorter than $80 \mathrm{~min}$ at $500^{\circ} \mathrm{C}$, indicating low oxidation resistance of these alloys. In contrast, alloying with calcium $(\mathrm{Ca})$, strontium $(\mathrm{Sr})$, beryllium, REs and silicon carbide $(\mathrm{SiC})$ nanoparticles suppress oxidation of magnesium alloys by extending the incubation period.

\subsection{Evaporation-enhanced magnesium oxidation}

The non-protective oxidation of magnesium alloys is also closely related to the high evaporation rate of magnesium at high temperatures, ${ }^{35,56}$ as the temperature approaches the melting temperature of the magnesium alloy. Magnesium exhibits a much higher tendency to evaporate during high-temperature oxidation than aluminium, titanium and iron $(\mathrm{Fe})$, as driven by the higher vapour pressure. For example, at $500^{\circ} \mathrm{C}$, the equilibrium vapour pressure of magnesium is $10 \cdot 2 \mathrm{~Pa}$, which is $1.9 \times 10^{11}, 5 \cdot 0 \times 10^{15}$ and $1.9 \times 10^{20}$ times larger than that of aluminium, iron and titanium, respectively. ${ }^{57}$ The magnesium vapour pressure also increases as the temperature exceeds the melting temperature of thermally unstable microconstituents, such as eutectic microstructures. ${ }^{32,33,58}$ The vigorous magnesium vapourisation contributes to the breakage of the surface oxide layer and to the accelerated oxidation. ${ }^{56}$

Figure 2 provides a schematic diagram of the evolution of the oxide layer on magnesium alloys during high-temperature oxidation. During the initial oxidation stage - that is, during the incubation period - the thin magnesium oxide layer is considered to be crack-free and therefore provides some protection to the alloy by isolating the substrate metal from oxygen, ${ }^{32,56}$ as shown in Figure 2(a). As the magnesium vapour diffusion is retarded by the continuous surface layer, oxidation occurs mainly by the ionic diffusion of magnesium ions $\left(\mathrm{Mg}^{2+}\right)$ through the surface layer,

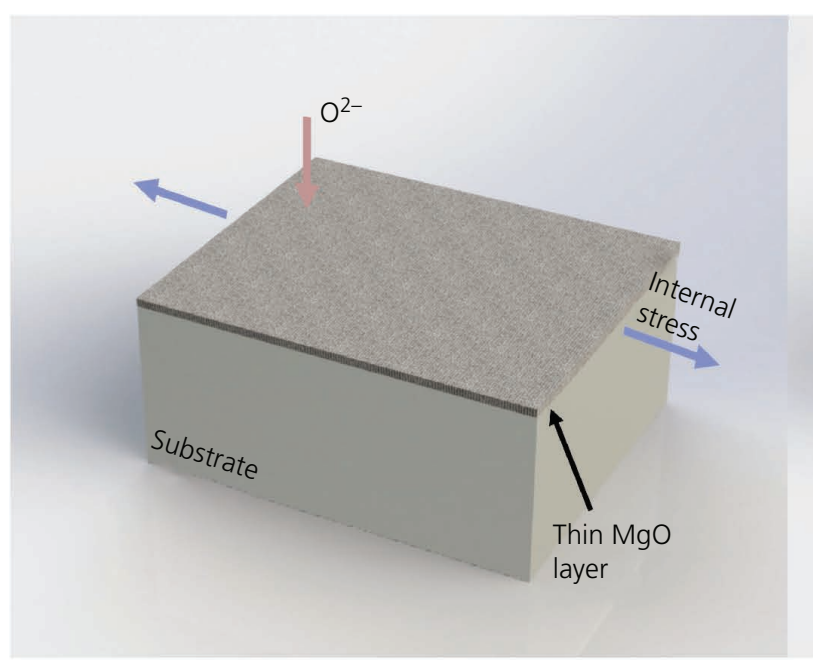

(a)

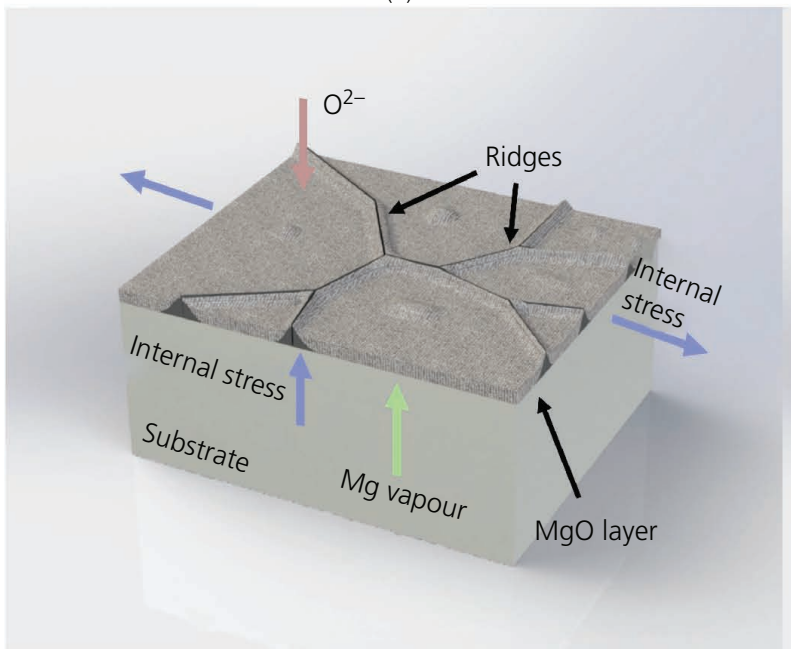

(c)

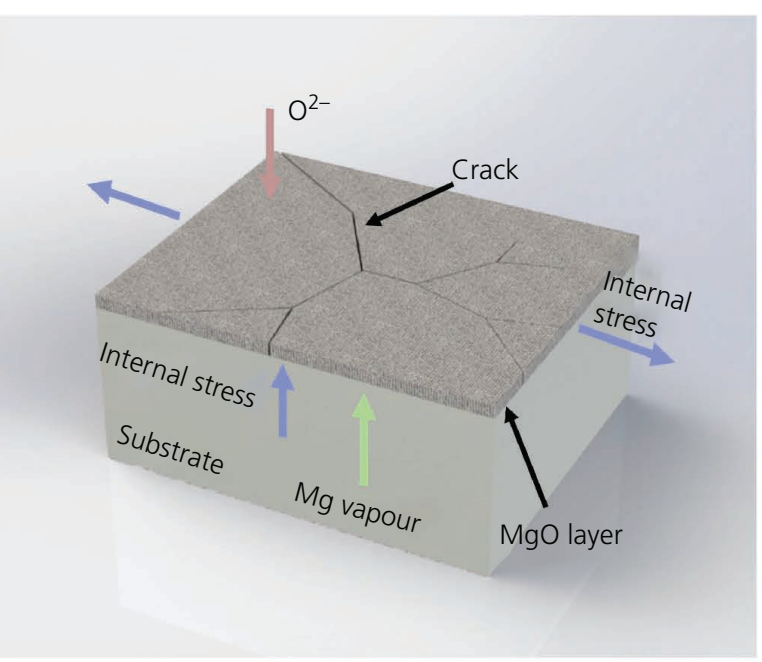

(b)

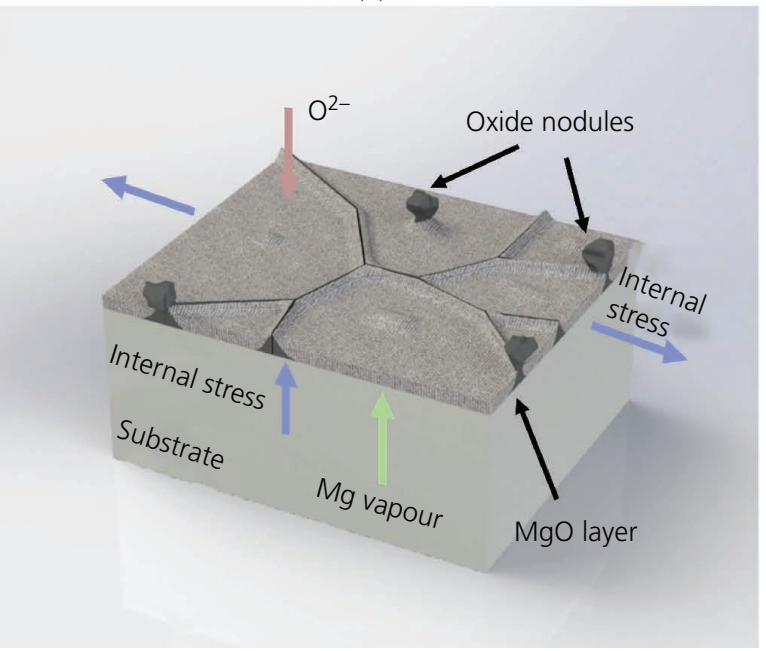

(d)

Figure 2. Schematic evolution of the oxide layer on magnesium alloys at high temperatures: (a) the formation of a thin magnesium oxide layer within the incubation period; (b) layer cracking within the magnesium oxide layer; (c) the formation of oxide ridges on the magnesium oxide layer; (d) nodular growth of the magnesium oxide at the outer surface 
which is driven by the activity gradient of magnesium ions across the oxide layer. ${ }^{28}$ This is a relatively slow process. ${ }^{59-61}$ However, due to the low $\mathrm{P}-\mathrm{B}$ ratio $\left(R_{\mathrm{PB}}\right)$ of magnesium oxide/magnesium of $0 \cdot 81$, an internal tensile stress is generated within the layer. ${ }^{20}$ Thickening of the magnesium oxide layer and the evaporation of underlying magnesium simultaneously cause the internal stress to increase, leading to the formation of cracks within the oxide layer, ${ }^{32,56}$ as illustrated in Figure 2(b). Thereafter, magnesium vapour and oxygen can easily transport through the cracks and react with each other to form porous magnesium oxide at the alloy/oxide layer interface, within the cracks and at the outer surface. ${ }^{27,62}$ As a result, the initially formed flat and continuous oxide layer is replaced by a ridge morphology, as shown in Figure 2(c). The vapour phase transport of magnesium is no longer restricted by the surface oxide layer, and the oxidation follows linear or power-law kinetics. ${ }^{13,30,32}$ As the non-protective oxidation continues, the growth of magnesium oxide exhibits a nodular morphology at the outer surface, as presented in Figure 2 (d). This is attributed to the rapid reaction between the magnesium vapour and oxygen at the outer surface..$^{59,62}$

Recently, Zhang et $a l^{63}$ further confirmed the evaporationenhanced mechanism of magnesium oxidation by studying the oxidation and evaporation behaviour of single-crystal magnesium nanoparticles at 130 and $200^{\circ} \mathrm{C}$ using an in situ environmental transmission electron microscope. An $\sim 2 \mathrm{~nm}$ thick preformed monocrystalline magnesium oxide layer had been formed on the sample surface prior to the oxidation test. The orientation relationship between the magnesium matrix and the magnesium oxide was $(0001)_{\mathrm{Mg}} \| \sim 2^{\circ}$ from $(1 \overline{1} 1)_{\mathrm{MgO}},[2 \overline{1} \overline{1} 0]_{\mathrm{Mg}} \|[001]_{\mathrm{MgO}} \cdot{ }^{63}$ The thin magnesium oxide surface film formed at ambient temperature was crystalline, in contrast to the previous assumption that amorphous magnesium oxide formed at room and at low temperatures. ${ }^{32,60}$ Increasing the temperature to 130 and $200^{\circ} \mathrm{C}$ led to a quick increase in the layer thickness and to the formation of magnesium oxide lamellae on top of the preformed oxide layer. After the initial stage, layer thickening was barely noticeable even after $243 \mathrm{~s}$ at $130^{\circ} \mathrm{C}$, indicating slow oxidation at this temperature. In contrast, irregular oxide bubbles formed at the magnesium/magnesium oxide interface after oxidation at $200^{\circ} \mathrm{C}$ for $12 \mathrm{~s} .{ }^{63}$ The bubble growth of the oxide layer was attributed to the intensified magnesium evaporation at $200^{\circ} \mathrm{C}$. Further oxidation resulted in oxide layer cracking and the subsequent growth of large magnesium oxide dendrites, indicating the occurrence of non-protective oxidation. ${ }^{63}$

\subsection{Effect of surface roughness}

An isothermal oxidation test in a TGA apparatus is often used as a standard method to characterise the high-temperature oxidation behaviour by measuring the specific weight gain $\left(\mathrm{mg} / \mathrm{cm}^{2}\right)$ of the specimen. ${ }^{28}$ The specimen area is typically taken to be the apparent area computed from the measured macroscopic specimen dimensions, without consideration of surface roughness. ${ }^{30,46,48,52}$ However, even a macroscopically smooth surface has a certain amount of surface roughness. ${ }^{64}$ Previous studies ${ }^{64-68}$ indicated that increasing specimen surface roughness significantly increased the initial oxidation rates of iron and nickel (Ni)-based superalloys.

The authors' recent work ${ }^{69}$ found that the initial oxidation rate of magnesium alloys also increases with increasing specimen surface roughness. Figure 3 presents the TGA results ${ }^{69}$ for the oxidation in air at $400^{\circ} \mathrm{C}$, for AZ91 specimens with increasing surface roughness $\left(R_{\mathrm{a}}\right)$ values of $0.05,0.49$ and $0.85 \mu \mathrm{m}$. The weight gain increased with increasing surface roughness. After oxidation at $400^{\circ} \mathrm{C}$ for $5 \mathrm{~h}$, the polished specimen, with $R_{\mathrm{a}}=0.05 \mu \mathrm{m}$, had the lowest overall weight gain of $0 \cdot 12 \mathrm{mg} / \mathrm{cm}^{2}$. The weight gain increased to $0.58 \mathrm{mg} / \mathrm{cm}^{2}$ with increasing $R_{\mathrm{a}}$ to $0.49 \mu \mathrm{m}$ and to $0.73 \mathrm{mg} / \mathrm{cm}^{2}$ for the specimen with $R_{\mathrm{a}}$ of $0 \cdot 85 \mu \mathrm{m}$. The increase in the surface roughness also led to a decrease in the incubation period. The incubation period of the polished specimen $\left(R_{\mathrm{a}}=0.05 \mu \mathrm{m}\right)$ was $\sim 150 \mathrm{~min}$, whereas the ground specimens with $R_{\mathrm{a}}$ values of 0.49 and $0.85 \mu \mathrm{m}$ exhibited an incubation period of $\sim 70 \mathrm{~min}$.

The higher oxidation rate of AZ91 with higher surface roughness was associated with the crests and grooves on the rougher surface. ${ }^{69}$ The crests and grooves facilitated oxidation at the initial stage by providing additional facets and sharp edges exposed to air for oxidation. ${ }^{65-68}$ In addition, the stress relaxation within the initially formed oxide layer was significantly restrained by the wavy geometry on the sharp edges, promoting layer cracking and the accelerated oxidation. ${ }^{28,65}$ Hence, compared with the polished specimen, the ground specimens with rougher surfaces exhibited higher oxidation rates and shorter incubation periods, as shown in Figure 3.

For magnesium oxidation, the specific weight gain represents the amount of oxygen absorbed into the oxide layer and is proportional to the layer thickness. ${ }^{28}$ In contrast, the incubation period indicates the protectability of the oxide layer and thereby the oxidation

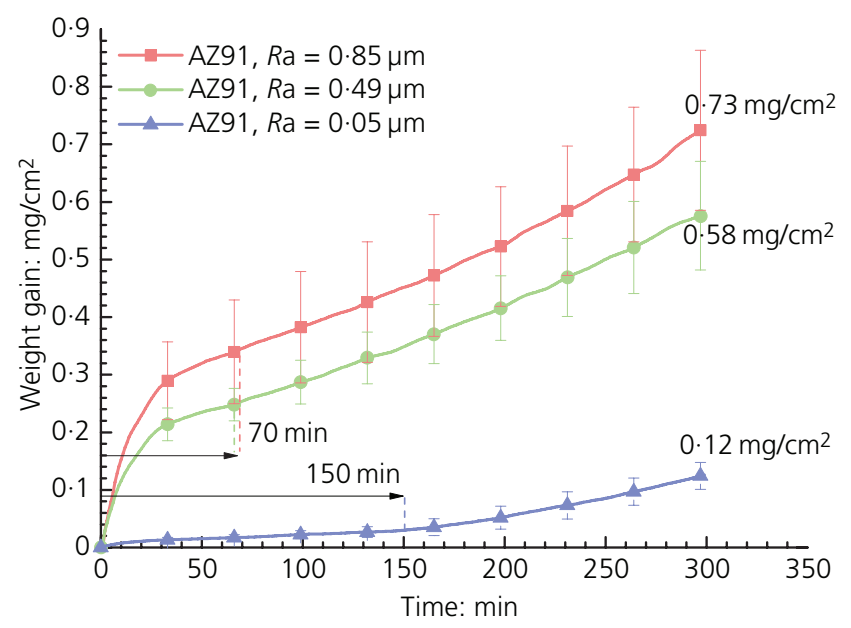

Figure 3. The isothermal oxidation kinetics of AZ91 in air at $400^{\circ} \mathrm{C}$ for $5 \mathrm{~h}$ for samples with different surface finishes. A higher surface roughness $\left(R_{a}\right)$ led to a more rapid oxidation rate at the initial oxidation stage. Reproduced from Tan et al. ${ }^{69}$ and used with permission from Elsevier 
resistance. ${ }^{56}$ The incubation period ends because cracking occurs in the initially formed magnesium oxide layer and non-protective fast oxidation begins. Reducing the surface roughness by 17 times led to a significant decrease in the weight gain but provided a relatively small extension to the incubation period. This indicated the low oxidation resistance of AZ91. Once the crests and grinding grooves on the outmost surface were completely consumed by oxidation, it would be expected that all specimens exhibit the same oxidation rate.

\section{Burning}

Continuing oxidation at a high temperature can result in ignition and subsequent burning of the magnesium alloy component or specimen. Similar to other metals, the burning of a magnesium alloy specimen can be understood by means of the concept of heat balance within the specimen during oxidation. ${ }^{70}$ During oxidation at a high temperature, heat can be input into the specimen by $(a)$ the external heat energy from heating in a furnace or in a flame and (b) the heat energy released by the exothermic oxidation. On the other hand, heat can be extracted from the magnesium alloy specimen by means of $(a)$ radiation, $(b)$ conduction, $(c)$ convection and $(d)$ melting and evaporation of the alloy. When the heat input exceeds the total heat extracted, exothermic oxidation becomes selfsustaining and ignition occurs. ${ }^{70}$ In contrast, burning extinguishes when heat extraction dominates. As mentioned, oxidation of magnesium alloys is accompanied by vigorous magnesium evaporation, particularly for semi-solid and molten conditions. The magnesium vapour significantly expands the contact area between oxygen and magnesium, promotes exothermic oxidation and favours ignition and subsequent burning. ${ }^{71}$ Hence, ignition of magnesium alloys is prone to occur at oxide layer cracks that are saturated with magnesium vapour. ${ }^{13,23}$ One typical feature of magnesium burning is the epitaxial growth of the oxide protuberances with a cauliflower morphology, which results from the outward diffusion of magnesium vapour, followed by direct reaction with oxygen at the oxide/air interface. ${ }^{23,27,29,72}$

\subsection{Ignition temperature}

The term 'ignition temperature' $\left(T_{\mathrm{i}}\right)$ represents the temperature at which a magnesium alloy initiates burning. ${ }^{12,73,74}$ The continuous heating test is used to measure the $T_{\mathrm{i}}$ of magnesium alloys because of its ease of use, its reproducibility for a particular experimental setup and its high precision, but there are large variations between different experimenters. ${ }^{75,76}$ In this test, the magnesium alloy specimen is heated in a furnace at a constant heating rate in a dry air atmosphere and the $T_{\mathrm{i}}$ is evaluated as the lowest temperature associated with a sharp rise in the specimen temperature due to the significant emission of heat after the onset of burning of the magnesium alloy. ${ }^{13,23,75}$ One should be aware that, although the $T_{\mathrm{i}}$ quantifies the burning resistance of a magnesium alloy, it is not an intrinsic parameter. ${ }^{23}$ Experimental conditions such as the specimen geometry, heating rate, air velocity, humidity and foreign metal contact all influence the measured $T_{\mathrm{i}}$ value. ${ }^{12,13,23}$ For example, the $T_{\mathrm{i}}$ of an AZ91 bulk specimen was measured to be $600^{\circ} \mathrm{C},{ }^{76}$ whereas AZ91 powders exhibited a much lower ignition temperature of $484^{\circ} \mathrm{C} .{ }^{77}$ Details on these factors influencing the $T_{\mathrm{i}}$ of magnesium alloys can be found in the paper of Tekumalla and Gupta. ${ }^{12}$

Figure 4 summarises published $T_{\mathrm{i}}$ data for magnesium alloys in the form of bulk specimens. ${ }^{35,37,38,50,52,74,76,78-101}$ The grid patterns on the columns indicate the $T_{\mathrm{i}}$ variations, which result from different

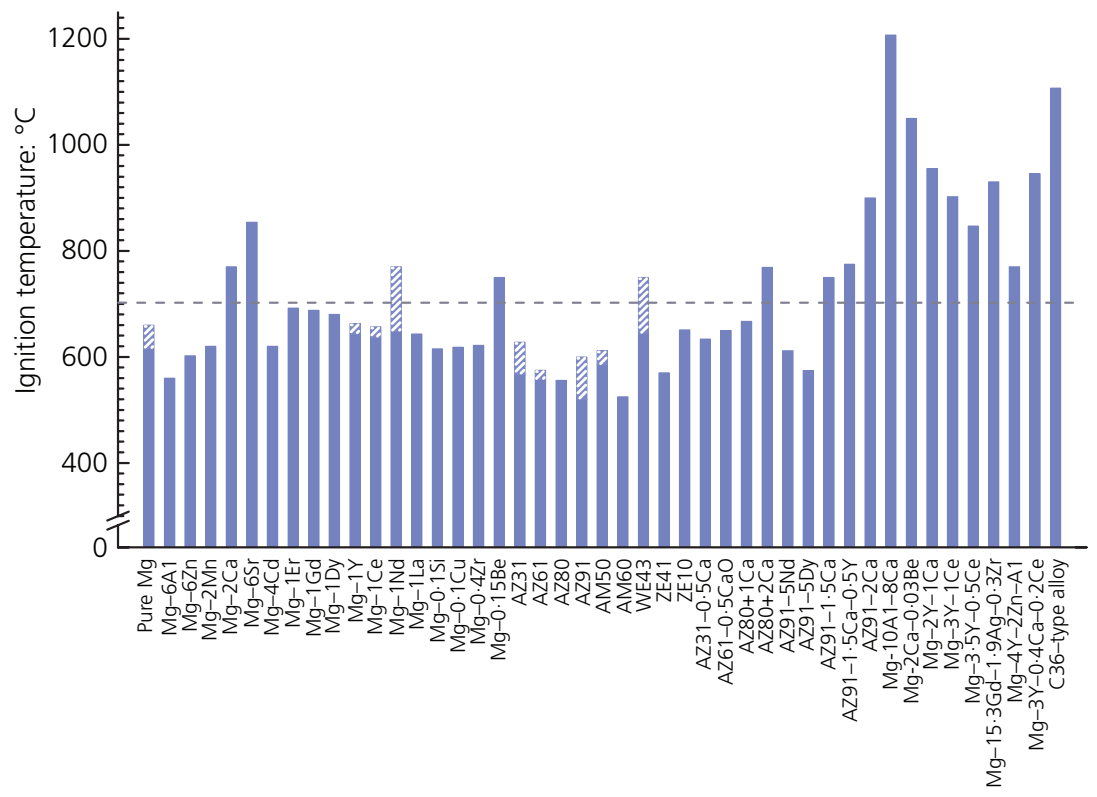

Figure 4. Ignition temperatures $\left(T_{\mathrm{i}}\right)$ of some magnesium alloys in the form of bulk from published data. ${ }^{35,37,38,50,52,74,76,78-101}$ The grid patterns in the columns indicate the temperature variations according to different tests. Aluminium, zinc, manganese, copper and silicon decrease the $T_{\mathrm{i}}$ of magnesium alloys, whereas calcium, strontium, beryllium and REs improve the $T_{\mathrm{i}}$. Alloys with high ignition temperatures over $700^{\circ} \mathrm{C}$, marked by the dashed line, are associated with high concentrations of calcium, strontium and REs of over 2 wt. \% 
testing conditions. Pure magnesium bulk ignites at temperatures between 615 and $660^{\circ} \mathrm{C}, 35,78-82$ which is close to or above its melting temperature of $650^{\circ} \mathrm{C} .{ }^{102}$ Figure 4 indicates that the alloying elements aluminium, zinc, manganese, copper and silicon ( $\mathrm{Si}$ ) decrease the $T_{\mathrm{i}}$ of magnesium alloys, attributed to the decrease in the solidus temperature and/or the formation of microconstituents with low melting temperatures with those solutes..$^{13,34,36,80,103}$ For example, alloying magnesium with aluminium leads to the formation of the magnesium- $-\mathrm{Mg}_{17} \mathrm{Al}_{12}$ eutectic microconstituent with a low melting temperature of $437^{\circ} \mathrm{C}$ along the grain boundaries of $\alpha$-magnesium. ${ }^{72,103-105}$ High-temperature oxidation causes preferential melting of the eutectic microconstituent, promoting magnesium evaporation along the grain boundaries and thereby leading to ignition of the alloy prior to bulk melting. ${ }^{13,27,33}$ Hence, commercial magnesium-aluminium-based alloys, such as AZ61, AZ91 and AM60, exhibit relatively low $T_{\mathrm{i}}$ as shown in Figure 4. Alloying elements that increase the $T_{\mathrm{i}}$ of magnesium alloys are calcium, strontium, beryllium and RE elements such as yttrium (Y),

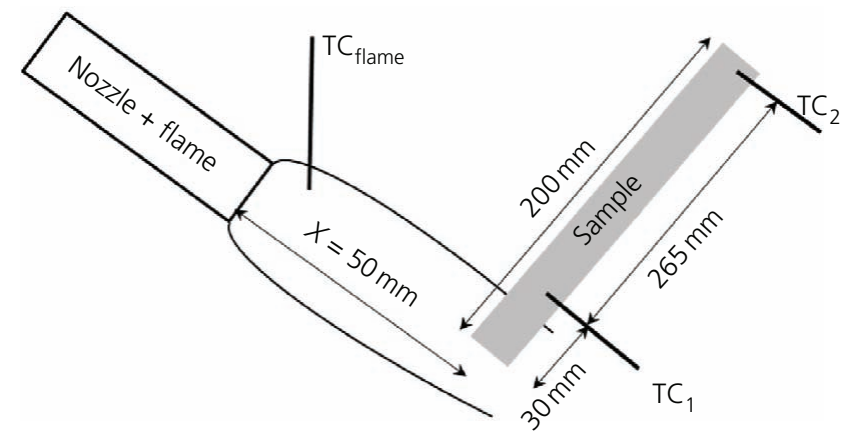

Figure 5. Schematic top view of the torch test. One end of the horizontal cylindrical magnesium alloy specimen was exposed to the flame from $L P G$ at $\sim 1190^{\circ} \mathrm{C}$, which was measured by a thermocouple $\left(\mathrm{TC}_{\text {flame }}\right)$ placed in the flame at a $50 \mathrm{~mm}$ distance from the mouth of the nozzle. The specimen was typically $20 \mathrm{~mm}$ in diameter and $200 \mathrm{~mm}$ in length. The embedded thermocouples $\left(\mathrm{TC}_{1}\right.$ and $\mathrm{TC}_{2}$ ) were used to monitor the temperature at the ends of each specimen. Reproduced from Prasad et al. ${ }^{90}$ with permission from Elsevier
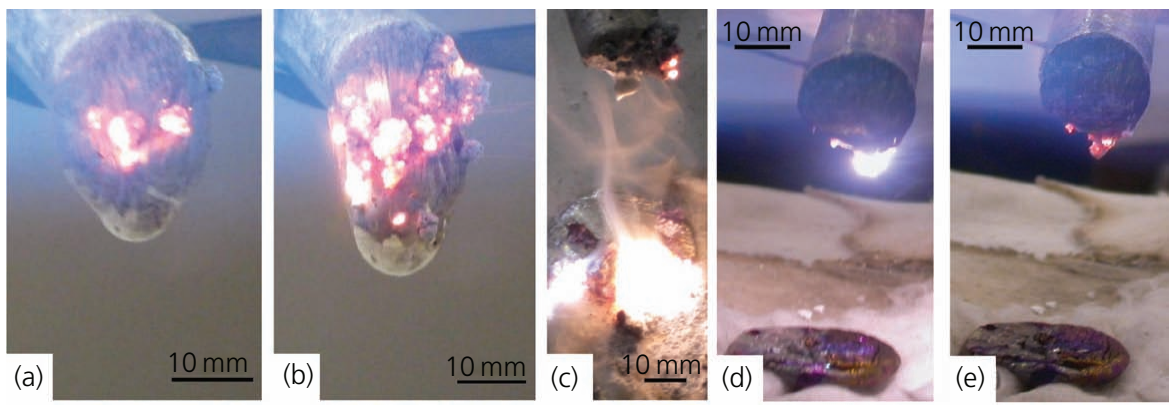

Figure 6. Burning behaviour of AZ61: (a) melting and partial ignition of the specimen tip after exposure to the LPG flame; (b) subsequent burning of the specimen tip; (c) burning of the detached blob after removal of the LPG flame. Burning behaviour of Mg-5Y: (d) melting and minor ignition of the specimen tip in the LPG flame; (e) extinguished ignition even in the presence of the LPG flame. Reproduced from Prasad et al. ${ }^{90}$ with permission from Elsevier

gadolinium (Gd) and neodymium (Nd). Such an increase is ascribed to the formation of a protective oxide layer on the alloy surface. ${ }^{13}$

Figure 4 indicates that the ignition-proof magnesium alloys with high $T_{\mathrm{i}}$ of over $700^{\circ} \mathrm{C}$ (marked by the horizontal dashed line) contain large amounts of calcium, strontium or REs of over $2 \mathrm{wt} . \%$. This leads to an increase in the alloy cost and significant embrittlement particularly in magnesium-calcium-based alloys. ${ }^{37,106-109}$ Thus, development of new ignition-proof magnesium alloys with lower concentrations of these alloying elements is of technological and practical significance.

\subsection{Flammability}

The term 'flammability' describes the tendency of a magnesium alloy specimen to burn when in contact with a flame or similar heat source and after heat source removal. ${ }^{12,13,23} \mathrm{~A}$ high resistance to flammability requires the alloy to self-extinguish easily when the heat source is removed. ${ }^{110}$

Prasad et al..$^{90,111}$ used a torch test as schematised in Figure 5 to study the flammability of high-purity (HP) magnesium, a range of magnesium $-X$ binary alloys $(X=$ aluminium, calcium, silicon, tin, strontium, lanthanum (La), manganese, zinc, zirconium ( $\mathrm{Zr}$ ), yttrium, cerium (Ce), gadolinium and neodymium) and commercial AZ61 and AZ91. The specimen tip was heated in a liquefied-petroleum-gas (LPG) flame until melted. For all studied alloys, burning started after some melting of the specimen tip. As melting of magnesium alloys significantly enhances magnesium evaporation, this result indicates that the magnesium vapour is the burning species for maintaining the flame. ${ }^{90,111}$

Figure 6 shows the typical burning behaviours of AZ61 and $\mathrm{Mg}-5 \mathrm{Y}$ using the torch test as illustrated in Figure 5. ${ }^{90}$ Figure 6(a) shows that the AZ61 tip melts and partially ignites after being subjected to the LPG flame for some time. Continuous heating in the LPG flame results in a more prominent burning of the specimen tip and the formation of oxide protuberances with cauliflower morphology on the attached molten blob, as illustrated in Figure 6(b). A blob of molten magnesium alloy often separates 
from the specimen tip after melting and burning for a period. When the LPG flame is removed, as shown by Figure 6(c), the burning of the specimen tip ceases, whereas the separated blob of molten AZ61 continues to burn until consumed completely. Similar results are also found for HP magnesium, AZ91 and aluminium-, silicon-, tin-, strontium-, manganese-, zinc- and zirconium-containing binary magnesium alloys, indicating the insufficient flammability resistance of those alloys. ${ }^{90,111}$

In contrast, for $\mathrm{Mg}-5 \mathrm{Y}$, there is only minor ignition at the specimen tip after melting, as shown in Figure 6(d). Then, burning self-extinguishes even in the presence of the LPG flame, as shown in Figure 6(e). In addition, the separated molten blob remains unignited within the entire test period. This result indicates that the $\mathrm{Mg}-5 \mathrm{Y}$ exhibited a considerable flammability resistance compared with AZ61, which is attributed to the formation of a protective yttrium-containing surface oxide layer. ${ }^{90}$ High flammability resistance is also found for calcium-, gadolinium-, cerium-, lanthanum- and neodymium-containing binary magnesium alloys, of which the detached molten blob does not burn during the test period. ${ }^{111}$ This result is consistent with the fact that alloying with calcium and REs significantly increases the $T_{\mathrm{i}}$ of magnesium alloys, as shown in Figure 4.

Similar observations were reported by Marker $^{25}$ from the FAA for commercially used magnesium alloys, including AZ31, AZ80, ZE41, ZE10, EV31, Elektron 675 (Mg-6Y-7Gd-0.5Zr) and WE43. Alloys with high concentrations of REs, typically WE43, EV31 and Elektron 675, exhibit a considerably higher tendency to self-extinguish after flame removal compared with other alloys. Geometrical factors, such as the specimen thickness, significantly affect the flammability resistance of magnesium alloys. ${ }^{25}$ The WE43 specimen with section thickness of 0.6 inch $(15.2 \mathrm{~mm})$ self-extinguishes instantaneously after flame removal, whereas the specimen with section thickness of 0.4 inch $(10.2 \mathrm{~mm})$ continues to burn for $\sim 2 \mathrm{~min}$. Hence, the geometric requirement in the component design is a significant factor determining the hightemperature applicability of magnesium alloys. Magnesium alloys with thin sections could be melted readily and have a higher tendency to ignite when contacting with sufficient heat.

\section{Theoretical models}

The low oxidation resistance of magnesium alloys has been attributed to $(a)$ the high vapour pressure of magnesium at elevated temperatures and $(b)$ the non-protective nature of the magnesium oxide layer. The high vapour pressure is a thermodynamic property of magnesium and is difficult to overcome owing to the low binding energy of magnesium atoms. ${ }^{112}$ The non-protective magnesium oxide layer has received considerable attention in the attempts to produce better protection through alloying magnesium alloys with the elements calcium, REs, strontium and beryllium. ${ }^{13,113}$

This section analyses $(a)$ the theoretical models that have been proposed for the mechanisms of how these alloying elements improve oxidation resistance and $(b)$ the applicability of the models to various oxidation-resistant magnesium alloys. The effects of alloying elements have been previously reviewed $^{12,13,23,60,74}$ and therefore are not discussed herein.

\subsection{P-B ratio}

Pilling and Bedworth ${ }^{20}$ indicated that the protectability of an oxide layer can be assessed by the volume ratio between the oxide formed and the corresponding metal consumed - that is, the $\mathrm{P}-\mathrm{B}$ ratio $\left(R_{\mathrm{PB}}\right)$

2. $R_{\mathrm{PB}}=\frac{V_{\text {ox }}}{V_{\mathrm{m}}}=\frac{M_{\text {oxide }} \rho_{\text {metal }}}{n M_{\text {metal }} \rho_{\text {oxide }}}$

where $V_{\mathrm{ox}}$ is the volume of the elementary cell of the metal oxide, $V_{\mathrm{m}}$ is the volume of the elementary cell of the corresponding metal, $M$ is the atomic or molecular mass, $n$ is the number of metal atoms in one molecule of the oxide, $\rho$ denotes density and the subscripts are self-explanatory.

In this model, $R_{\mathrm{PB}}<1$ indicates that tensile stresses are generated within the oxide so that the oxide layer has a high susceptibility to cracking due to the internal tensile stresses and is therefore expected to be non-protective. In contrast, $R_{\mathrm{PB}}>1$ indicates a complete coverage of the substrate by the oxide and a compressive stress within the oxide. However, $R_{\mathrm{PB}}>2$ indicates a large compressive stress that may cause oxide buckling and spalling, resulting in protection failure. ${ }^{20}$ Hence, the formation of a continuous surface oxide layer with $R_{\mathrm{PB}}$ between 1 and 2 is a critical factor in improving the high-temperature oxidation resistance of magnesium alloys. ${ }^{113}$

The $R_{\mathrm{PB}}$ value of magnesium oxide/magnesium is $0 \cdot 81$, indicating that the magnesium oxide layer has an incompact structure with an internal tensile stress.

Table 2 lists the $R_{\mathrm{PB}}$ values of the oxides of common alloying elements. ${ }^{13,20}$ The oxides aluminium oxide $\left(\mathrm{Al}_{2} \mathrm{O}_{3}\right)$, zinc oxide

Table 2. Commonly used alloying elements in magnesium alloys and the $R_{\mathrm{PB}}$ of their corresponding oxides ${ }^{13,20}$

\begin{tabular}{llc} 
Element & \multicolumn{1}{c}{ Oxide } & $\boldsymbol{R}_{\mathbf{P B}}$ \\
\hline Magnesium & Magnesium oxide & 0.81 \\
Aluminium & Aluminium oxide & 1.29 \\
Zinc & Zinc oxide & 1.59 \\
Silicon & Silicon dioxide & 1.89 \\
Calcium & Calcium oxide & 0.65 \\
Copper & Copper (I) oxide & 1.64 \\
Zirconium & Zirconium (IV) oxide & 1.56 \\
Strontium & Strontium oxide & 0.66 \\
Yttrium & Yttrium (III) oxide & 1.13 \\
Cerium & Cerium (III) oxide & 1.14 \\
& Cerium (IV) oxide & 1.15 \\
Neodymium & Neodymium (III) oxide & 1.13 \\
Gadolinium & Gadolinium (III) oxide & 1.23 \\
Lanthanum & Lanthanum (III) oxide & 1.11 \\
Beryllium & Beryllium oxide & 1.70
\end{tabular}


( $\mathrm{ZnO})$, silicon dioxide $\left(\mathrm{SiO}_{2}\right)$, copper (I) oxide $\left(\mathrm{Cu}_{2} \mathrm{O}\right)$, zirconium (IV) oxide $\left(\mathrm{ZrO}_{2}\right)$, beryllium oxide $(\mathrm{BeO})$ and all $\mathrm{RE}$ oxides exhibit $R_{\mathrm{PB}}$ values between 1 and 2 , indicating a possibly protective oxide.

Thermodynamics can be used to assess the conditions that can lead to the formation of a surface $X \mathrm{O}$ oxide on a given alloy magnesium $-X$. The possible reactions on the alloy surface during oxidation are ${ }^{13,28}$

\section{3. $2 \mathrm{Mg}+\mathrm{O}_{2}=2 \mathrm{MgO}$}

\section{4. $2 X+\mathrm{O}_{2}=2 X \mathrm{O}$}

The change in Gibbs free energy, $\Delta G$, of these reactions are ${ }^{13,28}$

$$
\begin{aligned}
\Delta G_{\mathrm{MgO}} & =\Delta G_{\mathrm{MgO}}^{0}+R T \ln \frac{\alpha_{\mathrm{MgO}}^{2}}{\alpha_{\mathrm{Mg}}^{2} p_{\mathrm{O}_{2}}} \\
& =\Delta G_{\mathrm{MgO}}^{0}+R T \ln \frac{1}{c_{\mathrm{Mg}}^{2} p_{\mathrm{O}_{2}}}
\end{aligned}
$$

$$
\begin{aligned}
\Delta G_{X \mathrm{O}} & =\Delta G_{X \mathrm{O}}^{0}+R T \ln \frac{\alpha_{X \mathrm{O}}^{2}}{\alpha_{X}^{2} p_{\mathrm{O}_{2}}} \\
& =\Delta G_{X \mathrm{O}}^{0}+R T \ln \frac{1}{c_{X}^{2} p_{\mathrm{O}_{2}}}
\end{aligned}
$$

where $\Delta G^{0}$ is the standard Gibbs free energy of formation, $T$ is the reaction temperature (in kelvins), $R$ is the gas constant, $\alpha$ represents the activity, $p$ is the partial pressure of oxygen and the subscripts are self-explanatory. Oxides, magnesium oxide and $X \mathrm{O}$ are regarded as pure solids, so both $\alpha_{\mathrm{MgO}}$ and $\alpha_{X \mathrm{O}}$ are equal to $1 .{ }^{114}$ For metals, $\alpha_{\mathrm{Mg}}$ and $\alpha_{X}$ can be approximated by their mole atomic concentrations $c_{\mathrm{Mg}}$ and $c_{X}$, respectively. ${ }^{13,28}$

The thermodynamic feasibility of the formation of $X \mathrm{O}\left(\Delta G_{X \mathrm{O}}<\right.$ $\Delta G_{\mathrm{MgO}}{ }^{28}$ ) can be represented as

7. $\frac{c_{\mathrm{Mg}}}{c_{X}}<\exp \frac{\Delta G_{\mathrm{MgO}}^{0}-\Delta G_{X \mathrm{O}}^{0}}{2 R T}$

In binary alloys, $c_{\mathrm{Mg}}$ can be expressed by $1-c_{X}$, yielding

8. $\frac{1}{c_{X}}<\exp \frac{\Delta G_{\mathrm{MgO}}^{0}-\Delta G_{X \mathrm{O}}^{0}}{2 R T}+1$

Equation 8 can be satisfied for $(a)$ a highly negative value of $\Delta G_{X \mathrm{O}}^{0}$ and $(b)$ a high concentration of $X, c_{X}$, on the alloy surface.
The first point is determined by the chemical activity of the alloying element $X$, while the second point requires a substantial accumulation of $X$ on the alloy surface, which is closely related to the maximum solubility of $X$ in magnesium. ${ }^{12,28} \mathrm{Kim}$ et al. ${ }^{113}$ suggested that an alloying element that may improve the oxidation resistance of a magnesium alloy requires $(a)$ a high solubility limit (>2 at.\%) in magnesium, $(b)$ a high chemical reactivity and (c) an appropriate $R_{\mathrm{PB}}$ value (between 1 and 2).

This model allows understanding of the improved oxidation resistance of most RE-containing magnesium alloys. ${ }^{21,39,115-117}$ REs such as yttrium, gadolinium, dysprosium (Dy) and erbium (Er) have high solid solubility ( $>2$ at. $\%$ ) in magnesium so that surface accumulation can be easily achieved during oxidation, leading to the formation of an $\mathrm{RE}$ oxide layer with the appropriate $R_{\mathrm{PB}}$ on the alloy surface to isolate the alloy from oxygen. Yu et al. ${ }^{21}$ reported a dense, compact and continuous yttrium (III) oxide $\left(\mathrm{Y}_{2} \mathrm{O}_{3}\right)$ layer $\left(R_{\mathrm{PB}}=1 \cdot 3\right)$ on the surface of a $\mathrm{Mg}-5 \cdot 5 \mathrm{Y}$ alloy that protected the alloy from oxidation at $600^{\circ} \mathrm{C}$ for over $100 \mathrm{~min}$. Wu et al. ${ }^{100}$ developed a non-flammable $\mathrm{Mg}-15 \cdot 3 \mathrm{Gd}-1 \cdot 9 \mathrm{Ag}-0 \cdot 3 \mathrm{Zr}$ (GQ152K) alloy that was able to withstand a flame at $930^{\circ} \mathrm{C}$ for over $6 \mathrm{~min}$. The high flammability resistance was attributed to the coverage of the entire alloy surface by a gadolinium (III) oxide $\left(\mathrm{Gd}_{2} \mathrm{O}_{3}\right)$ layer $\left(R_{\mathrm{PB}}=1 \cdot 23\right)$. Similar results were reported for magnesiumneodymium, magnesium-dysprosium and magnesium-erbium in both solid and molten conditions. ${ }^{35,93,113}$

Although this model allows understanding of the oxidation resistance of RE-containing magnesium alloys, it is not applicable to alloys such as magnesium-calcium, magnesium-strontium and in particular magnesium-beryllium. Calcium oxide $(\mathrm{CaO}) /$ calcium and strontium oxide $(\mathrm{SrO}) /$ strontium have low $R_{\mathrm{PB}}$ values of 0.65 and 0.66 , which should indicate a highly incompact layer. ${ }^{20}$ Moreover, the solid solubilities of calcium and strontium in magnesium are limited to 0.65 and 0.66 at.\%, respectively, indicating difficulty in obtaining sufficient surface accumulation of both solutes. ${ }^{113}$ However, alloying with calcium and/or strontium significantly improves the oxidation resistance and increases the $T_{\mathrm{i}}$ of magnesium alloys. ${ }^{36,38,46,84,92,118}$ For magnesium-beryllium, although the solid solubility of beryllium is almost zero in magnesium, ${ }^{103}$ alloying with beryllium at parts per million (ppm) concentrations greatly enhances the oxidation resistance of magnesium alloys. ${ }^{83,114,119-121}$ Houska $^{119}$ showed an increase of $200^{\circ} \mathrm{C}$ in the $T_{\mathrm{i}}$ of pure magnesium through alloying with $10 \mathrm{ppm}(\mathrm{wt})$ beryllium. Czerwinski ${ }^{120}$ reported a decrease in the oxidation rate at $487^{\circ} \mathrm{C}$ of $\mathrm{AZ91}$ containing $10 \mathrm{ppm}$ (wt) beryllium. Owing to the high affinity of beryllium to oxygen and the appropriate $R_{\mathrm{PB}}$ value of beryllium oxide/beryllium (1.70), such an improvement has long been ascribed to the formation of a continuous protective beryllium oxide layer on the alloy surface. $^{20,121}$ However, beryllium oxide has not been observed. Moreover, the authors' previous work ${ }^{45}$ confirmed that the formation of beryllium oxide on magnesium alloys was not thermodynamically feasible at ppm concentrations. Thus, this model needs refinement. 


\subsection{Surface-active elemental effect}

Surface-active elements, $X_{\mathrm{Sa}}$, are solutes that decrease the surface energy and surface tension of a liquid metal and exhibit a tendency to segregate to the melt surface. ${ }^{122,123}$ Such surface segregation alters the surface composition, evaporation behaviour and oxidation rate of the liquid metal, resulting in the 'surfaceactive elemental effect' (SAEE). ${ }^{13,84}$ Yamauchi ${ }^{124}$ proposed that in a binary substitutional-solid solution, the solute element with a larger Wigner-Seitz radius, $r_{\mathrm{s}}$, tends to segregate to the surface of the solvent metal and to reduce the surface energy. Figure 7 presents the $r_{\mathrm{s}}$ values of some alloying elements in magnesium, ${ }^{84,125}$ which implies that sodium (Na), calcium, cerium, neodymium, tin and strontium are surface-active elements in a magnesium melt because their $r_{\mathrm{s}}$ values are larger than that of magnesium.

Improving the oxidation resistance of magnesium alloys by way of strontium alloying is an example of an SAEE. ${ }^{46,84,126,127}$ Aydin et al. ${ }^{84}$ found a progressive increase in $T_{\mathrm{i}}$ with increasing strontium concentration for magnesium-strontium alloys. $\mathrm{Mg}-6 \mathrm{Sr}$ exhibited the highest $T_{\mathrm{i}}$ of $854^{\circ} \mathrm{C}$, which was $200^{\circ} \mathrm{C}$ higher than that of pure magnesium. $\mathrm{Mg}-6 \mathrm{Sr}$ could be melted directly in air without burning even after isothermal holding at $700^{\circ} \mathrm{C}$ for $12 \mathrm{~h}$. Both X-ray diffraction and X-ray photoelectron spectroscopy detected a significant surface segregation of strontium oxide after oxidation, attributed to the SAEE of strontium in the magnesium melt. Hence, the improved oxidation resistance was attributed to the dense continuous strontium oxide-rich layer on the $\mathrm{Mg}-6 \mathrm{Sr}$ melt. ${ }^{46,84}$ Aydin et $a l .{ }^{84}$ considered that, despite the small $R_{\mathrm{PB}}$ value of strontium oxide/strontium of $0 \cdot 66$, any crack within the oxide layer was promptly healed through selective oxidation of strontium owing to the surface enrichment of strontium and its

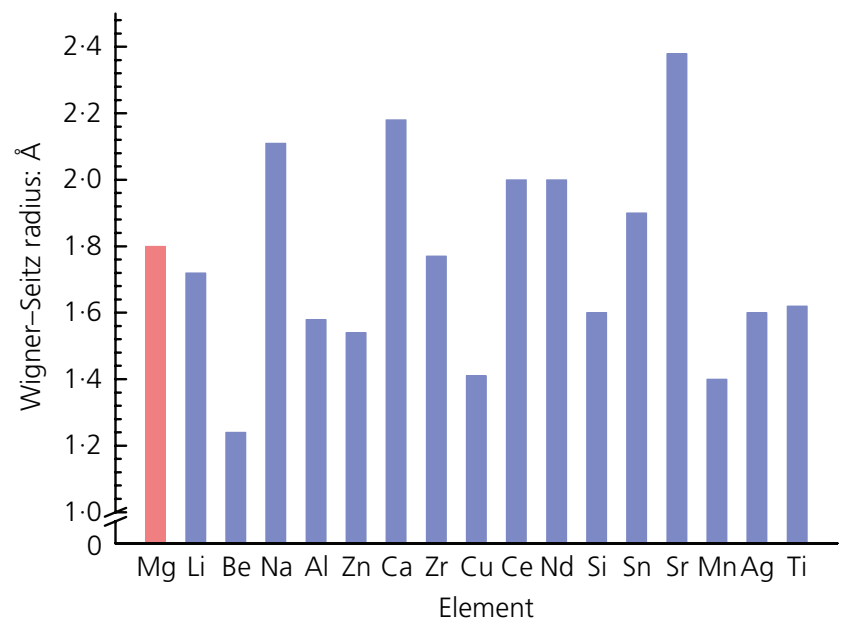

Figure 7. Wigner-Seitz radius, $r_{s}$, of some elements. Elements including sodium, calcium, cerium, neodymium, tin and strontium have the potential to segregate to the surface of magnesium melt owing to their $r_{\mathrm{s}}$ values being larger than that of magnesium. Data retrieved from Aydin et al. ${ }^{84}$ and Ren et al. ${ }^{125}$ high affinity to oxygen. In comparison, alloying with strontium did not significantly increase the oxidation resistance of magnesium in the solid state. ${ }^{46,84}$ This was attributed to the insufficient surface accumulation of strontium through sluggish diffusion in the solid state. ${ }^{13}$

Many studies have reported $^{52,53,87,92,118,128,129}$ that calcium improves the ignition resistance of magnesium alloys by forming a calcium oxide-rich oxide layer. However, as mentioned, calcium oxide/calcium exhibits a low $R_{\mathrm{PB}}$ value of $0 \cdot 65$, indicating a nonprotective calcium oxide. ${ }^{20}$ Hence, the protective capacity of calcium oxide is hard to reconcile with the $R_{\mathrm{PB}}$ model. In analogy with strontium and the high $r_{\mathrm{s}}$ value of calcium $(\sim 2 \cdot 2 \AA$ in Figure 7), the improved ignition resistance of calcium-containing magnesium alloys can be attributed to the SAEE of calcium. After melting of the alloy, surface accumulation of calcium is easily achieved due to the SAEE, promoting the formation of calcium oxide to heal any cracks within magnesium oxide. ${ }^{129}$ Despite the low $R_{\mathrm{PB}}$ of calcium oxide/calcium, the formation of a continuous calcium oxide-magnesium oxide composite layer effectively suppresses magnesium vapourisation and thereby increases $T_{\mathrm{i}}$. Unlike strontium, calcium also improves the oxidation resistance of magnesium alloys in the solid state. ${ }^{36,37,129}$ The authors' recent study $^{129}$ further confirmed the formation of a continuous calcium oxide-rich oxide layer on AZ91 containing $1 \mathrm{wt} \%$ calcium that significantly decreased the oxidation rate of the alloy at $400^{\circ} \mathrm{C}$. Since the SAEE was marginal in the solid state, the outward diffusion of calcium ions $\left(\mathrm{Ca}^{2+}\right)$ through the magnesium oxide grain boundaries was the only channel enabling the formation of calcium oxide. The present authors consider that calcium oxide may segregate within the magnesium oxide grain boundaries, resulting in either a reinforcement effect to the magnesium oxide or the reactive element effect (REE) (more details are provided in section 4.3). Experimental evidence is required to check these hypotheses.

The protective oxide layer forming on strontium- and calciumcontaining magnesium alloys indicated that the $R_{\mathrm{PB}}$ is not the only criterion for selecting proper alloying elements to improve the oxidation resistance of magnesium alloys. Due to the surface segregation of strontium and calcium in the magnesium melt, the formation of strontium oxide and calcium oxide is significantly facilitated, protecting the melt from oxidation despite their inappropriate $R_{\mathrm{PB}}$ values. Thus, alloying magnesium alloys with surface-active elements appears to be a promising approach for ignition-proof applications.

Figure 7 shows that sodium, cerium, neodymium and tin also tend to segregate to the magnesium melt surface because of the high $r_{\mathrm{s}}$ values. It would be fruitful to study the SAEE of these alloying elements in magnesium melts and therefore develop new ignitionresistant magnesium alloys.

\subsection{Reactive element effect}

The term 'reactive element effect' relates to the influence of minor alloying with a reactive element $\left(X_{\mathrm{Re}}\right)$ with high affinity to 
oxygen (such as REs, zirconium, titanium and calcium) on enhancing the protective nature of the surface oxide. ${ }^{60}$ This concept was first proposed by Pfeil $^{130}$ for a cerium-containing nickel-chromium (Cr) alloy, where alloying with cerium, a reactive element with a high affinity to oxygen, enhanced the protective nature of the surface nickel (II) oxide (NiO) layer. The REE of other elements such as yttrium and lanthanum has also been subsequently reported in nickel, chromium, and aluminium alloys. $^{131-133}$ Czerwinski and Szpunar ${ }^{134}$ and Czerwinski ${ }^{135}$ confirmed the REE mechanism of cerium in nickel (II) oxide, wherein the segregation of cerium (IV) ions $\left(\mathrm{Ce}^{4+}\right)$ along the nickel (II) oxide grain boundaries effectively inhibited the diffusion of nickel (II) ions $\left(\mathrm{Ni}^{2+}\right)$ and therefore reduced the oxidation rate by two orders of magnitude.

Although there is no experimental evidence confirming the REE during magnesium oxidation at present, this possible mechanism was proposed by Czerwinski ${ }^{32}$ in analogy with the REE in nickel (II) oxide, chromium (III) oxide $\left(\mathrm{Cr}_{2} \mathrm{O}_{3}\right)$ and aluminium oxide. Figure 8 schematically portrays the REE mechanism on magnesium oxidation. During high-temperature oxidation, particles of the $X_{\mathrm{Re}}$ oxides $\left(X_{\mathrm{Re}} \mathrm{O}\right)$ along the magnesium oxide grain boundaries decompose and populate the magnesium oxide grain boundaries with $X_{\mathrm{Re}}{ }^{2+}$ through the following reaction ${ }^{32}$

9. $X_{\mathrm{Re}} \mathrm{O}+\mathrm{Mg}^{2+}=X_{\mathrm{Re}}{ }^{2+}+\mathrm{MgO}$

During the early oxidation stage, the growth of the magnesium oxide layer is dominated by the outward diffusion of magnesium cations, followed by reaction with superoxide anions $\left(\mathrm{O}^{2-}\right)$ at the oxide/air interface. ${ }^{136}$ The segregation of $X_{\mathrm{Re}}{ }^{2+}$ cations along the magnesium oxide grain boundaries effectively hinders the grain boundary diffusion of magnesium cations, ${ }^{60}$ as illustrated in Figure 8. As a consequence, at a sufficiently high $X_{\mathrm{Re}}$ concentration, the growth of the magnesium oxide is primarily governed by the inward diffusion of superoxide anions along the magnesium oxide grain boundaries, which is too slow to enable a substantial oxidation rate. ${ }^{32}$ Because the decrease in the ionic diffusion of magnesium ions requires a concentration of $X_{\mathrm{Re}}{ }^{2+}$

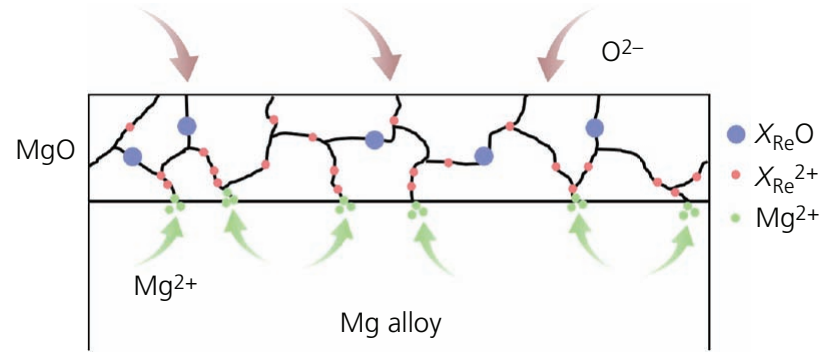

Figure 8. Schematic diagram of the proposed REE mechanism on magnesium oxidation. The $X_{\operatorname{Re}}{ }^{2+}$ in magnesium oxide grain boundaries block the diffusion of magnesium cations along the magnesium oxide grain boundaries, a higher $X_{\mathrm{Re}}$ concentration is expected to cause a lower oxidation rate.

In addition, the outward diffusion of magnesium cations leads to an inward vacancy flux, creating voids at the magnesium oxide/ magnesium interface and thereby facilitating layer cracking. ${ }^{13}$ The present authors consider that the suppression of ionic diffusion of magnesium ion through the REE may also contribute to extending the protective incubation period. Further investigation is needed to test this hypothesis.

Based on this analogy, an REE tendency has been suggested for REs, calcium and beryllium in magnesium alloys. ${ }^{38,39,120}$ Wang et al. $^{39}$ reported a decreased oxidation rate of pure magnesium at both 400 and $500^{\circ} \mathrm{C}$ after yttrium implantation (yttrium ions of $90 \mathrm{keV}$ with a dose of $5 \times 10^{17} \mathrm{~cm}^{-2}$ for $1 \mathrm{~h}$ ). The improved oxidation resistance was attributed to a dense compact surface oxide consisting of magnesium oxide and yttrium (III) oxide. In addition, there were scattered nodules with a high elemental oxygen $(\mathrm{O})$ content on the surface of the implanted sample. Wang et $a .^{39}$ ascribed the oxide nodules to the segregation of yttrium ions along the grain boundaries of magnesium oxide, similar to the REE of cerium in nickel (II) oxide. ${ }^{134}$ However, experimental evidence is required to check for the segregation of yttrium ions. A more recent study by $\mathrm{Lee}^{38}$ reported an increase in the oxidation resistance and $T_{\mathrm{i}}$ of AZ31 after alloying with $0.3 \mathrm{wt} . \%$ calcium. Their transmission electron microscopy (TEM) indicated that the improved oxidation resistance resulted from the formation of a protective magnesium oxide layer which incorporated calcium cations. However, it was unclear whether the calcium ions segregated along the magnesium oxide grain boundaries and provided an REE or reacted with the element oxygen to form a calcium oxide barrier layer. Further investigation is required to clarify this mechanism. A similar result was found in a berylliumcontaining AZ91 alloy by Czerwinski. ${ }^{120}$ Microalloying with $10 \mathrm{ppm}$ (wt) beryllium caused a noticeable decrease in the oxidation rate of $\mathrm{AZ91}$ at $487^{\circ} \mathrm{C}$ by suppressing magnesium evaporation. Czerwinski ${ }^{120}$ attributed the mechanism to the REE of beryllium. However, no experimental evidence was provided to support this hypothesis.

\section{Evaluation of these models}

The $\mathrm{P}-\mathrm{B}$ ratio, $R_{\mathrm{PB}}$, uses the volume ratio between the oxide and the corresponding metal to provide an indirect estimation of the surface oxide layer compactness and the stress within the oxide layer. However, this model is qualitative and is limited to the characterisation of the protectability of a continuous oxide such as an RE oxide layer on a magnesium alloy. This model does not explain how the alloying elements calcium, strontium and beryllium improve the oxidation resistance of magnesium alloys.

The SAEE indicates that a surface-active element, $X_{\mathrm{Sa}}$, segregates to the magnesium melt surface to form $X_{\mathrm{Sa}} \mathrm{O}$ and thereby may form a more protective $X_{\mathrm{Sa}} \mathrm{O}$-magnesium oxide composite oxide layer. ${ }^{84}$ This model can explain the high efficiency of calcium and 
strontium in improving the ignition resistance of magnesium alloys. However, this model is only qualitative. Validation of this model requires a quantitative relationship between the surfaceactive element and the protectability of the $X_{\mathrm{Sa}} \mathrm{O}$-magnesium oxide layer, such as the layer integrity and strength.

The REE model is different from the prior two models. The REE model suggests that the $X_{\operatorname{Re}}{ }^{2+}$ ions segregate along the grain boundaries of the native magnesium oxide and inhibit the ionic diffusion of the native metal (magnesium ion), instead of forming a separate barrier layer. ${ }^{60}$ The key feature is that a minor or trace amount of alloying can decrease the oxidation rate significantly. ${ }^{60}$ This model appears to be applicable for strontium and beryllium, which exhibit high efficiencies in improving the oxidation resistance of magnesium even at ppm concentration. ${ }^{119,120,126}$ However, validation of this model requires precise characterisation of the oxide and, in particular, the oxide grain boundary composition.

These existing models have been frequently used in the literature to rationalise the oxidation resistance of magnesium alloys. However, these models are only qualitative and do not correlate the improved oxidation resistance with the crack resistance of surface oxide layers.

A new approach is the realisation that the oxidation resistance of magnesium alloys is closely related to the mechanical properties of the surface oxide. A surface oxide with a higher mechanical strength is expected to be able to withstand a higher internal tensile stress without cracking, delaying the onset of non-protective oxidation. This new approach is presented in the next section.

\section{Beryllium and the OR model}

Previous research ${ }^{45,55,69,129}$ elucidated (a) the effect of trace beryllium additions on the high-temperature oxidation of cast magnesium alloys, (b) the microstructure of the surface oxide using TEM and $(c)$ the mechanical properties of the oxide using nanoindentation results. The OR model was proposed for the oxidation resistance of magnesium alloys. Details of this research are reviewed in this section.

\subsection{Oxidation behaviour of beryllium-containing AZ91} Prior research ${ }^{45,69,129}$ showed that trace alloying of beryllium (10-60 ppm (wt)) into AZ91 significantly decreases the oxidation rate at $400^{\circ} \mathrm{C}$ and increases the $T_{\mathrm{i}}$ of $\mathrm{AZ91}$. The oxidation resistance increases with increasing beryllium content.

Figure 9 shows typical isothermal oxidation kinetics of AZ91 and AZ91Be60 (containing $60 \mathrm{wt}$. ppm beryllium) at $400^{\circ} \mathrm{C}$ for 300 min, determined by TGA. ${ }^{69}$ AZ91 had an oxidation incubation period of $150 \mathrm{~min}$ at $400^{\circ} \mathrm{C}$, after which there was accelerated oxidation with a rapid weight gain. In comparison, oxidation of AZ91Be60 followed parabolic kinetics for the entire testing period, indicating an incubation period of over $300 \mathrm{~min}$. After oxidation at $400^{\circ} \mathrm{C}$ for $300 \mathrm{~min}$, the overall specific weight gain of AZ91 was $0 \cdot 118 \mathrm{mg} / \mathrm{cm}^{2}$, but that for AZ91Be60 was only $0.016 \mathrm{mg} / \mathrm{cm}^{2}$, a

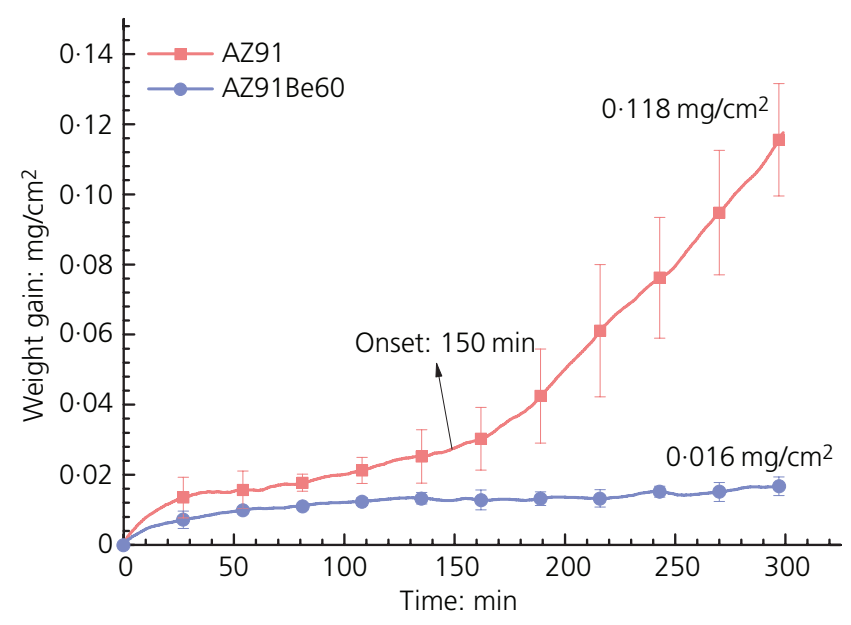

Figure 9. The isothermal oxidation kinetics of AZ91 and AZ91Be60 at $400^{\circ} \mathrm{C}$ in air for $5 \mathrm{~h}$. Reproduced from Tan et al. ${ }^{69}$ with permission from Elsevier

decrease by $\sim 7$ times. This result indicates that microalloying with beryllium improves the oxidation resistance of AZ91 by effectively extending the protective incubation period.

Figure 10 shows macrographs of AZ91 and beryllium-containing AZ91 after long-term furnace oxidation (LTFO) at $400^{\circ} \mathrm{C}$ in air for $144 \mathrm{~h}$ and after removing the surface oxide. ${ }^{45}$ AZ91 was massively destroyed after the test, attributed to the catastrophic oxidation, signifying a non-protective oxide layer on the specimen surface. $^{29}$ In comparison, all beryllium-containing alloys had much less oxidation. This indicates the formation of highly protective oxide layers on the beryllium-containing AZ91. Alloying AZ91 with $60 \mathrm{ppm}$ (wt) beryllium also increased the $T_{\mathrm{i}}$ from 549 to $659^{\circ} \mathrm{C} .{ }^{129}$

\section{$6.2(\mathrm{Mg}, \mathrm{Be}) \mathrm{O}$ layer}

The initial oxide is crack-free on both AZ91 and AZ91Be60, has a similar thickness of $\sim 100 \mathrm{~nm}$ (Figures 11(a) and 11(e)) and consists of nanosized grains (Figures 11(b) and 11(f)). ${ }^{69}$ The grain size of the oxide on AZ91Be60 is much smaller than that on AZ91, indicating grain refinement of the initially formed oxide layer through beryllium addition. The polycrystalline nature of the oxides is confirmed by the selected area diffraction (Saed) patterns (Figures 11(c) and 11(g)). Each oxide is composed of face-centred cubic magnesium oxide, and there is no beryllium oxide, as evidenced by indexing the diffraction patterns. The energy-dispersive spectroscopy (EDS) spectra (Figures 11(d) and 11(h)) show large magnesium and oxygen peaks within both oxide layers, confirming the formation of magnesium oxide. In addition, the oxide on AZ91Be60 contains beryllium, as evidenced by the noticeable beryllium peak in the EDS spectrum (Figure 11(h)) and the absence of such a beryllium peak for the AZ91 oxide (Figure 11(d)). In beryllium-containing magnesium alloys, beryllium is considered to mainly segregate along the grain boundaries of $\alpha$-magnesium owing to limited solid solubility of 


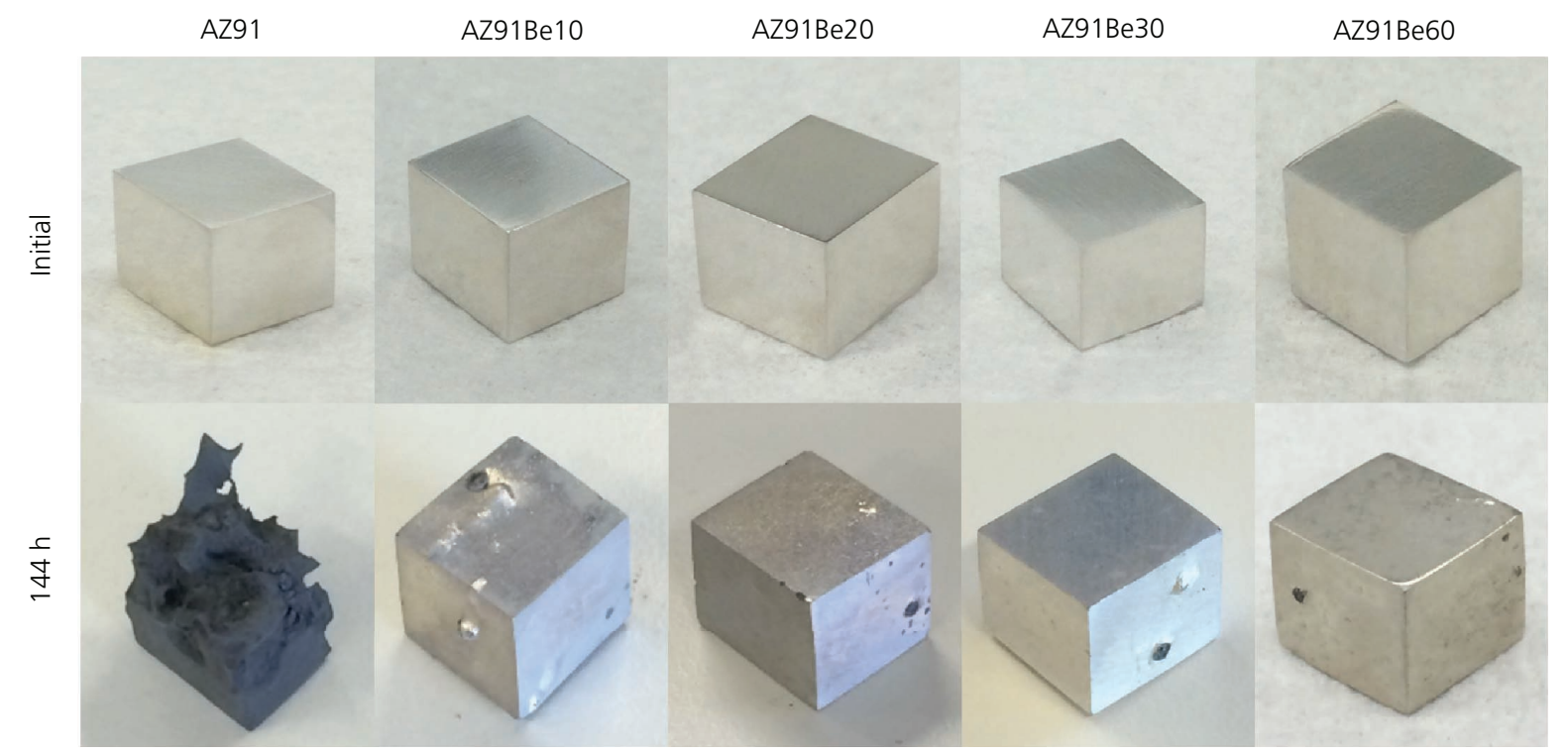

Figure 10. The macrographs of AZ91 and beryllium-containing AZ91 alloys after oxidation at $400^{\circ} \mathrm{C}$ for $144 \mathrm{~h}$. Reproduced from Tan et al. ${ }^{45}$ with permission from Elsevier

almost zero in magnesium. ${ }^{45,55,103}$ Hence, during the hightemperature oxidation, the surface enrichment of beryllium is achieved through ionic diffusion of beryllium ions $\left(\mathrm{Be}^{2+}\right)$ along the grain boundary of $\alpha$-magnesium, forming the berylliumenriched magnesium oxide layer. ${ }^{69}$

The lattice parameters $a_{\mathrm{MgO}}$ for the magnesium oxide and $a_{\mathrm{Be}-\mathrm{MgO}}$ for the beryllium-enriched magnesium oxide are $4.293 \AA$ and $4 \cdot 201 \AA$, respectively. ${ }^{69}$ The beryllium enrichment leads to a decrease in the lattice parameter of the magnesium oxide by $2 \cdot 14 \%$, attributed to the formation of $(\mathrm{Mg}, \mathrm{Be}) \mathrm{O}$ substitutional solid solution, where the magnesium ion was partially substituted by beryllium ions, causing lattice distortion. ${ }^{69,121,137}$

\subsection{Reinforcement effect}

The hardness $H$ of both magnesium oxide and $(\mathrm{Mg}, \mathrm{Be}) \mathrm{O}$ layers was measured using nanoindentation and nanoscratch tests on the surfaces of AZ91 and AZ91Be60 after $2 \mathrm{~h}$ TGA oxidation at $400^{\circ} \mathrm{C}$. The strength is expected to be related proportionally to the hardness.

Figure 12 presents fitting of the measured hardness values, $H$, to the hardness of the oxide and substrate through a power-law function as follows ${ }^{69,138}$

10. $H=H_{\mathrm{s}}\left(\frac{H_{1}}{H_{\mathrm{s}}}\right)^{1 /[1+A(h / d) B]}$

where $H_{\mathrm{s}}$ and $H_{1}$ are the hardness of the substrate and the oxide layer, respectively; $h$ represents the indenter penetration depth; $d$ denotes the layer thickness, determined by the TEM; and $A$ and
$B$ are adjustable coefficients. The dashed line is the substrate hardness $H_{\mathrm{s}}$. The best fits to Equation 10 , as presented by the solid curves, yield $H_{1}=1.5 \mathrm{GPa}$ for magnesium oxide and $H_{1}=$ $4 \cdot 1 \mathrm{GPa}$ for $(\mathrm{Mg}, \mathrm{Be}) \mathrm{O}$ at the boundary condition of $h / d \rightarrow 0$. This indicates a significant reinforcement by beryllium on the initially formed $(\mathrm{Mg}, \mathrm{Be}) \mathrm{O}$.

The significantly higher strength of $(\mathrm{Mg}, \mathrm{Be}) \mathrm{O}$ compared with that of magnesium oxide is confirmed by nanoscratch tests. ${ }^{69}$ The critical scratch force causing cracking of the layer is $296 \mu \mathrm{N}$ for magnesium oxide and $413 \mu \mathrm{N}$ for $(\mathrm{Mg}, \mathrm{Be}) \mathrm{O}$, confirming the higher resistance of the $(\mathrm{Mg}, \mathrm{Be}) \mathrm{O}$ layer against layer cracking.

The increased mechanical strength and hardness of $(\mathrm{Mg}, \mathrm{Be}) \mathrm{O}$ indicates significant reinforcement by beryllium on the initially formed magnesium oxide. The reinforcement is attributed to both solid solution strengthening and grain refinement strengthening in the $(\mathrm{Mg}, \mathrm{Be}) \mathrm{O}$ layer. ${ }^{69}$ The lattice distortion within $(\mathrm{Mg}, \mathrm{Be}) \mathrm{O}$ owing to the substitution of magnesium ions with beryllium ions increases the energy barrier against dislocation movement, causing solid solution hardening. ${ }^{139-141}$ In addition, the $(\mathrm{Mg}, \mathrm{Be}) \mathrm{O}$ layer with refined oxide grains provided more grain boundaries that impede the movement of dislocations. ${ }^{46,142,143}$ As a result, the $(\mathrm{Mg}, \mathrm{Be}) \mathrm{O}$ layer exhibits a higher mechanical strength to resist layer cracking.

As mentioned before, the oxidation resistance of magnesium alloys is closely related to the oxidation incubation period, which is mainly governed by the protectiveness of the initially formed oxide layer. Layer cracking and debonding facilitate the evaporation of magnesium, causing catastrophic oxidation, ignition and burning of magnesium alloys. ${ }^{69}$ The formation of the 

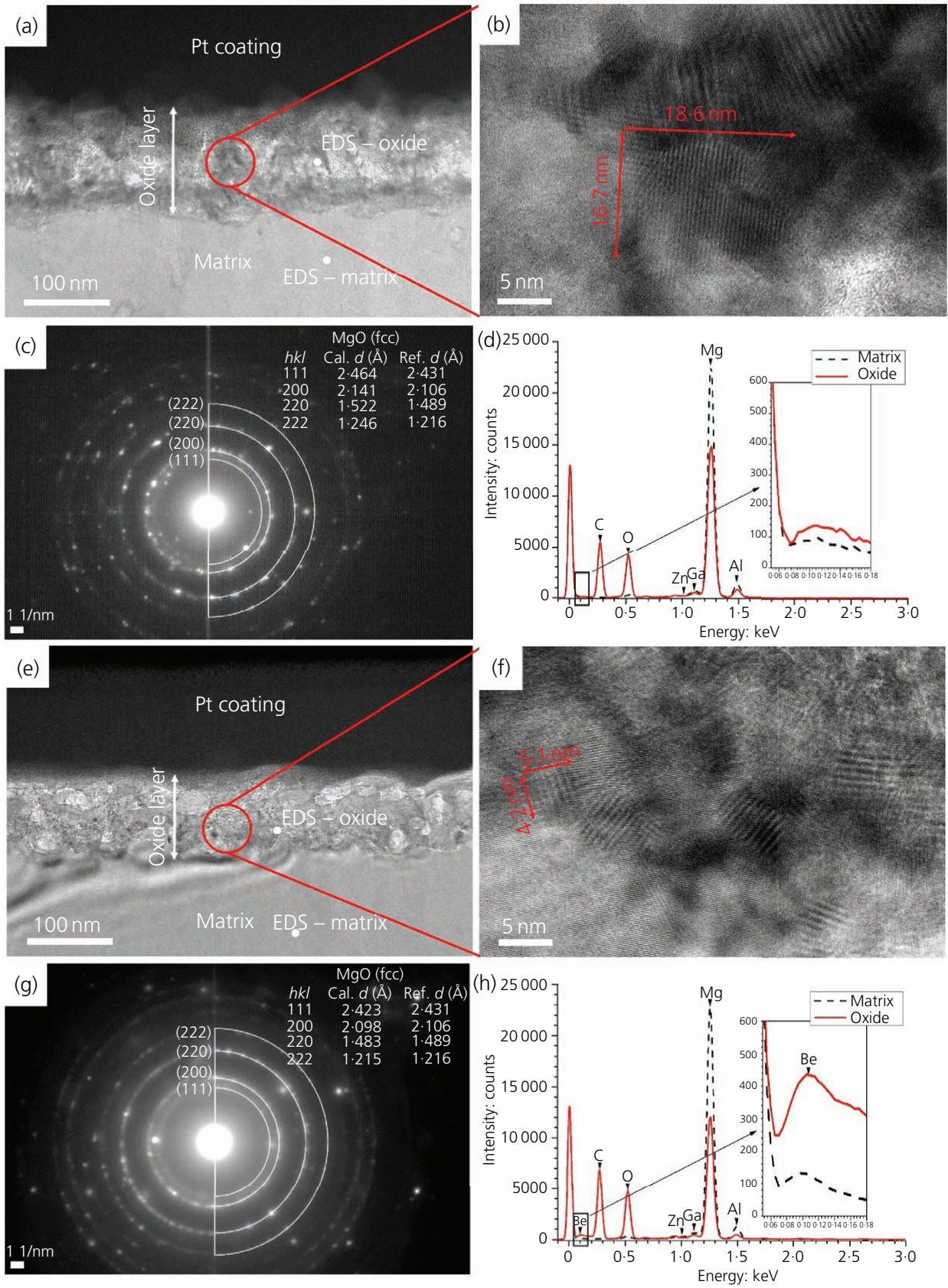

Figure 11. Cross-sectional TEM bright-field images, high-resolution TEM images, Saed pattern, and compositional analysis by way of EDS of the oxide layers formed on (a-d) AZ91 and (e-h) AZ91Be60, respectively, after $2 \mathrm{~h}$ oxidation at $400^{\circ} \mathrm{C}$ in air. The values of the calculated interplanar distance (Cal. $d$ ) and the reference interplanar distance (Ref. $d$ ) for magnesium oxide (retrieved from Joint Committee on Powder Diffraction Standards card no. 4-0829) are included in (c) and (g). Reproduced from Tan et al. ${ }^{69}$ with permission from Elsevier

fine-grained $(\mathrm{Mg}, \mathrm{Be}) \mathrm{O}$ solid solution provides higher mechanical strength and hardness of the oxide layer to withstand the internal tension stresses arising during oxidation. Therefore, layer cracking and the vapour phase transport of magnesium are effectively delayed, causing an extension in the oxidation incubation period of the AZ91Be60, as shown in Figure 9. As a result, the oxidation is mainly controlled by the outward diffusion of magnesium cations through the $(\mathrm{Mg}, \mathrm{Be}) \mathrm{O}$ layer, which is too slow to allow a substantial oxidation rate. ${ }^{69}$

The OR model is supported by the experimental findings $(a)$ that beryllium is incorporated into the magnesium oxide solid solution to form $(\mathrm{Mg}, \mathrm{Be}) \mathrm{O}$ and $(b)$ that $(\mathrm{Mg}, \mathrm{Be}) \mathrm{O}$ has a higher hardness. This means that the OR model can explain the better oxidation 


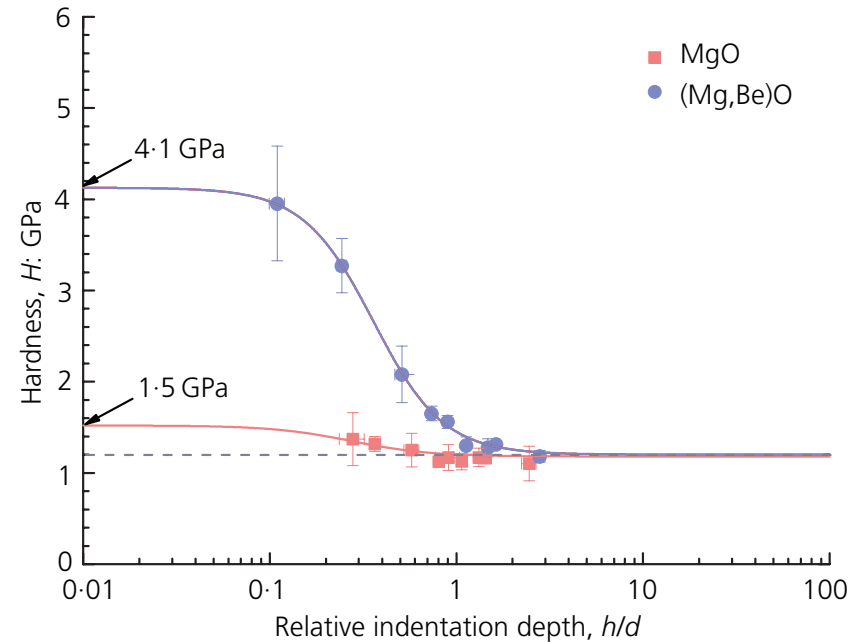

Figure 12. The hardness values of each oxide as a function of the relative indentation depth $h / d$. The substrate hardness is represented by the dashed line. The solid curves were best fits of Equation 10. The actual hardness value of each oxide is derived from the curves at $h / d \rightarrow 0$. Reproduced from Tan et al. ${ }^{69}$ with permission from Elsevier

resistance of beryllium-containing magnesium alloys. In addition, the incorporation of beryllium into $(\mathrm{Mg}, \mathrm{Be}) \mathrm{O}$ does mean that there is beryllium potentially available at the oxide grain boundaries to inhibit grain boundary diffusion of magnesium ion in the oxide.

\subsection{Generalisation of the OR model}

In order to generalise the OR model, $60 \mathrm{ppm}$ (wt) beryllium was microalloyed into magnesium alloys: $\mathrm{Mg}-2 \mathrm{Zn}, \mathrm{Mg}-2 \mathrm{Sn}, \mathrm{Mg}-2 \mathrm{Y}$, AS21, AM60, ZK20 and ZC63. ${ }^{55}$ TGA, LTFO and continuous heating tests indicated that microalloying with $60 \mathrm{ppm}$ (wt) beryllium effectively lowered the oxidation rates at $500^{\circ} \mathrm{C}$ and increased the $T_{\mathrm{i}}$ of $\mathrm{Mg}-2 \mathrm{Zn}, \mathrm{Mg}-2 \mathrm{Sn}, \mathrm{AS} 21$ and ZC63. $\mathrm{Mg}-2 \mathrm{Zn}-\mathrm{Be}, \mathrm{Mg}-2 \mathrm{Sn}-\mathrm{Be}$ and $\mathrm{AS} 21 \mathrm{Be}$ exhibited incubation periods over $300 \mathrm{~min}$ at $500^{\circ} \mathrm{C}$ and $T_{\mathrm{i}}$ values either close to or slightly above their respective liquidus temperatures, demonstrating the high ignition resistance of these alloys through microalloying with beryllium. ${ }^{55,103,144}$ Similarly, $\mathrm{Mg}-2 \mathrm{Y}$ exhibited a slow oxidation rate at $500^{\circ} \mathrm{C}$ and a high $T_{\mathrm{i}}$ of $647^{\circ} \mathrm{C}$, similar to the oxidation behaviour of $\mathrm{Mg}-2 \mathrm{Y}-\mathrm{Be}$ (containing $60 \mathrm{ppm}$ (wt) Be). ${ }^{55}$ All of these alloys had high hardness values of their initially formed oxide layers (Figure 13). ${ }^{55}$ Microalloying with $60 \mathrm{ppm}$ (wt) significantly reinforced the oxide on $\mathrm{Mg}-2 \mathrm{Zn}-\mathrm{Be}, \mathrm{Mg}-2 \mathrm{Sn}-\mathrm{Be}, \mathrm{AS} 21 \mathrm{Be}$ and $\mathrm{ZC} 63 \mathrm{Be}$ by increasing the hardness values by over two times. Similarly, the oxide layer on $\mathrm{Mg}-2 \mathrm{Y}$ exhibited the highest hardness value of $10 \cdot 2 \mathrm{GPa}$ among these alloys, and there was still a high hardness after alloying with beryllium of $8 \cdot 1 \mathrm{GPa}$ for $\mathrm{Mg}-2 \mathrm{Y}-\mathrm{Be}$.

In comparison, beryllium alloying provided a small influence on the oxidation resistance of ZK20 and AM60. ZK20Be and

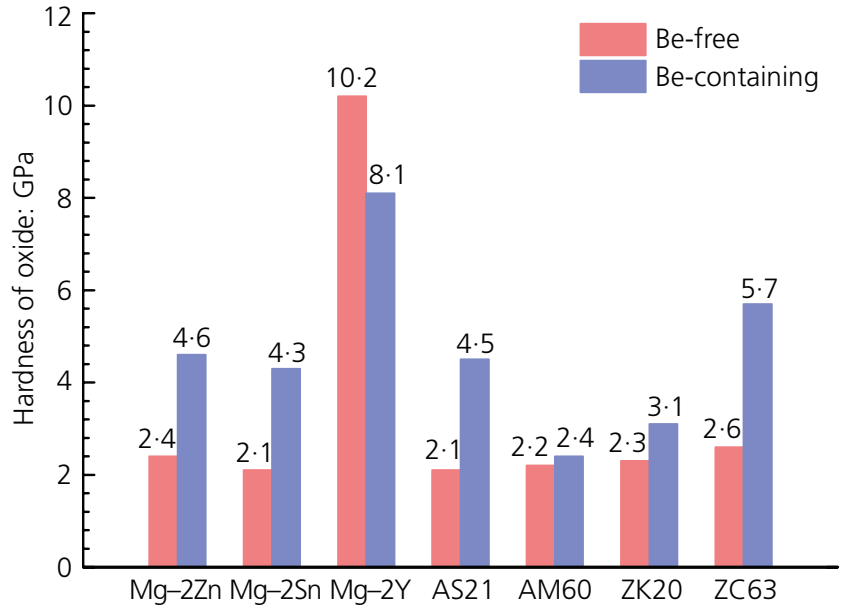

Figure 13. The hardness values of the initially formed oxide layers on Mg-2Zn, Mg-2Y, Mg-2Sn, AS21, AM60, ZK20 and ZC63 with and without beryllium, measured by nanoindentation tests. Data retrieved from Tan et al. ${ }^{55}$ with permission from Elsevier

AM60Be exhibited relatively short incubation periods of 60 and $20 \mathrm{~min}$ at $500^{\circ} \mathrm{C}$, respectively. Their $T_{\mathrm{i}}$ values were marginally increased by less than $10^{\circ} \mathrm{C}$ compared with those of the counterpart alloys without beryllium. In agreement with the OR model, Figure 13 shows that the reinforcement effect was relatively small in ZK20Be and AM60Be.

These results confirmed that the oxidation resistance of magnesium alloys is closely related to the hardness of the initially formed oxide layer. ${ }^{55}$ The high oxidation resistance of $\mathrm{Mg}-2 \mathrm{Zn}-\mathrm{Be}, \mathrm{Mg}-2 \mathrm{Sn}-\mathrm{Be}, \mathrm{Mg}-2 \mathrm{Y}, \mathrm{Mg}-2 \mathrm{Y}-\mathrm{Be}, \mathrm{AS} 21 \mathrm{Be}$ and $\mathrm{ZC} 63 \mathrm{Be}$ is attributed to their surface oxide layers with high hardness values, which provided sufficient strength to delay layer cracking and therefore extended the protective incubation period. In contrast, there were insufficient reinforcement in the initially formed oxide layers on AM60Be and ZK20Be, so that both alloys exhibited low oxidation resistance.

In analogy with AZ91Be60, the reinforcement effect on $\mathrm{Mg}-2 \mathrm{Zn}-\mathrm{Be}, \mathrm{Mg}-2 \mathrm{Sn}-\mathrm{Be}, \mathrm{AS} 21 \mathrm{Be}$ and ZC63Be was ascribed to the $(\mathrm{Mg}, \mathrm{Be}) \mathrm{O}$ solid solution. ${ }^{55}$ This is consistent with the fact that each of these alloys exhibited a significantly extended incubation period and an increased $T_{\mathrm{i}}$ higher than its liquidus temperature. The TEM results shown in Figure 11 indicated that the surface accumulation of beryllium is the necessary condition to form $(\mathrm{Mg}, \mathrm{Be}) \mathrm{O}$ during oxidation. Inductively coupled plasma emission spectroscopy and field emission scanning electron microscopy (FESEM) showed that the zirconium and manganese solutes in ZK20Be and AM60Be chemically reacted with the alloyed beryllium, forming thermally stable beryllium-containing intermetallic compounds that arrested beryllium to diffuse outwards. ${ }^{55}$ Therefore, the reinforcement effect was dramatically weakened. This suggested the incompatibility of beryllium with zirconium and manganese in improving the oxidation resistance of magnesium alloys. 
Both $\mathrm{Mg}-2 \mathrm{Y}$ and $\mathrm{Mg}-2 \mathrm{Y}-\mathrm{Be}$ exhibit high oxidation resistance and high oxide hardness values, indicating the same OR mechanism for the yttrium-containing magnesium alloys. The authors attributed such a significant OR effect to the formation of the yttrium (III) oxide layer $\left(R_{\mathrm{PB}}=1 \cdot 13\right){ }^{48,55,85}$ The somewhat decreased oxide hardness on $\mathrm{Mg}-2 \mathrm{Y}-\mathrm{Be}$ was attributed to the influence of redundant aluminium introduced by the aluminium-5 wt.\% beryllium master alloy during the alloy production. ${ }^{55}$ FESEM showed the existence of thermostable $\mathrm{Al}_{2} \mathrm{Y}$ within $\mathrm{Mg}-2 \mathrm{Y}-\mathrm{Be}$, which consumed yttrium and resulted in less yttrium (III) oxide on the alloy surface. ${ }^{145}$ Hence, despite the formation of $(\mathrm{Mg}, \mathrm{Be}) \mathrm{O}$, the reinforcement effect of yttrium was weakened, resulting in the decrease in the oxide hardness on $\mathrm{Mg}-2 \mathrm{Y}-\mathrm{Be}$. This also suggests that alloying with $2 \mathrm{wt} . \%$ yttrium provided greater reinforcement of the oxide on magnesium alloy compared with $60 \mathrm{ppm}$ (wt) beryllium.

\subsection{Evaluation of the OR model}

The OR model differs from the previous models and quantifies the protective capacity of an oxide using the mechanical strength/ hardness determined by nanoindentation and nanoscratch techniques. It has been shown that the oxidation resistance of magnesium alloys is closely related to the strength/hardness of the initially formed oxide layer. The formation of an oxide layer with higher mechanical strength and hardness is accompanied by a higher resistance to layer cracking and therefore an extended incubation period of the alloy.

The improved oxidation resistance of beryllium-containing magnesium alloys has been well explained by the reinforcement effect of beryllium on the initially formed magnesium oxide layer. TEM, nanoindentation and nanoscratch analyses provided evidence confirming the surface accumulation of beryllium and the formation of a fine-grained $(\mathrm{Mg}, \mathrm{Be}) \mathrm{O}$ solid solution with improved mechanical strength and hardness during the incubation period. Thus, the $(\mathrm{Mg}, \mathrm{Be}) \mathrm{O}$ layer exhibits a high protective capacity against cracking during oxidation, resulting in high oxidation resistance. However, the $(\mathrm{Mg}, \mathrm{Be}) \mathrm{O}$ structure has not been fully understood yet. Further investigation on the $(\mathrm{Mg}, \mathrm{Be}) \mathrm{O}$ structure at the atomic level - that is, the distribution of beryllium within the oxide layer - is needed to achieve an in-depth understanding of the reinforcement mechanism of berylliumcontaining magnesium oxide layer.

The nanoindentation and nanoscratch techniques provide new approaches to understanding the oxidation resistance of magnesium alloys and to quantifying the protectability of the surface oxide. Based on the OR effect of beryllium and yttrium, it would be reasonable to expect that the OR effect would be valid for other elements such as calcium, strontium and REs in improving the oxidation resistance of magnesium alloys. Thus, further investigations are needed in this area to generalise the OR model in other magnesium alloy systems. Such studies will provide significant breakthroughs in developing alloys particularly for applications at elevated temperatures.

\section{Synergistic effect of beryllium and calcium}

Considering the cost and toxic nature of beryllium, the usage of beryllium has long been expected to be minimised in magnesium alloys. ${ }^{23,37,146,147}$ The authors' recent work ${ }^{129}$ investigated the combined effect of beryllium and calcium on improving the oxidation resistance of magnesium alloys, trying to replace beryllium partially with calcium in oxidation-resistant magnesium alloys. Beryllium- and calcium-containing AZ91 alloys were produced by alloying beryllium (10, 20 and $60 \mathrm{ppm}(\mathrm{wt}))$ and calcium $(0.5$ and $1.0 \mathrm{wt} . \%)$ into alloy melts. Both TGA and LTFO tests, and the determined $T_{\mathrm{i}}$, evidenced that the combined addition of beryllium and calcium provided better protection to AZ91 than single addition of either beryllium or calcium.

Figure 14 shows the macroscopic surface appearances of the alloys after the LTFO at $400^{\circ} \mathrm{C}$ in air for $144 \mathrm{~h} .{ }^{129}$ Similar to the result presented in Figure 10, AZ91 was massively consumed by the catastrophic oxidation during the LTFO test, attributed to the nonprotective nature of the magnesium oxide layer. Compared with AZ91, alloying with either calcium or beryllium resulted in less oxidation for AZX910 (0.5 wt.\% calcium), AZX911 (1.0 wt.\% calcium) and in particular AZ91Be60 (60 ppm (wt) beryllium) after the LTFO test, indicating an increase in oxidation resistance. However, the formation of black scales on AZX910 and AZX911 and oxide nodules on AZ91Be60 indicated insufficient protections of their surface oxide layer against long-term oxidation at high temperatures. $^{13,58,62}$ In comparison, AZX910Be20 ( $0 \cdot 5$ wt. $\%$ calcium and $20 \mathrm{ppm}$ (wt) beryllium), AZX911Be10 (1.0 wt.\% calcium and $10 \mathrm{ppm}$ (wt) beryllium), AZX911Be20 (1.0 wt.\% calcium and $20 \mathrm{ppm}$ (wt) beryllium), AZX910Be60 (0.5 wt.\% calcium and $60 \mathrm{ppm}(\mathrm{wt})$ beryllium) and AZX911Be60 (1.0 wt.\% calcium and $60 \mathrm{ppm}$ (wt) beryllium) showed no noticeable oxidation after the LTFO test, signifying the high oxidation resistance of these alloys. This result demonstrated that combined alloying with beryllium and calcium achieves better oxidation resistance of AZ91 compared with single alloying with either beryllium or calcium.

The synergistic effect of beryllium and calcium was also manifested in the ignition resistance of AZ91. ${ }^{129}$ AZ91 had a $T_{\mathrm{i}}$ of $549^{\circ} \mathrm{C}$. Alloying with either $60 \mathrm{ppm}$ (wt) beryllium or $1.0 \mathrm{wt} . \%$ calcium significantly increased the $T_{\mathrm{i}}$ by $\sim 110$ to $\sim 660^{\circ} \mathrm{C}$. In comparison, with the combined addition of $20 \mathrm{ppm}$ (wt) beryllium and $0.5 \mathrm{wt} . \%$ calcium, AZX910Be20 exhibited a higher $T_{\mathrm{i}}$ of $677^{\circ} \mathrm{C}$. This result indicated that $0.5 \mathrm{wt} . \%$ calcium addition enabled a remarkable reduction in the beryllium usage in AZ91 by over $60 \%$ owing to the synergistic effect of beryllium and calcium. Further addition of either calcium or beryllium resulted in higher $T_{\mathrm{i}}$. AZX911Be60, containing $60 \mathrm{ppm}$ (wt) beryllium and 1.0 wt.\% calcium, exhibited the highest $T_{\mathrm{i}}$ of $697^{\circ} \mathrm{C}$ among all studied alloys.

The FESEM results indicated that the synergistic effect of beryllium and calcium enabled the formation of a compact duplex oxide layer on AZX911Be60, which was composed of the outer reinforced magnesium oxide layer and the inner calcium 


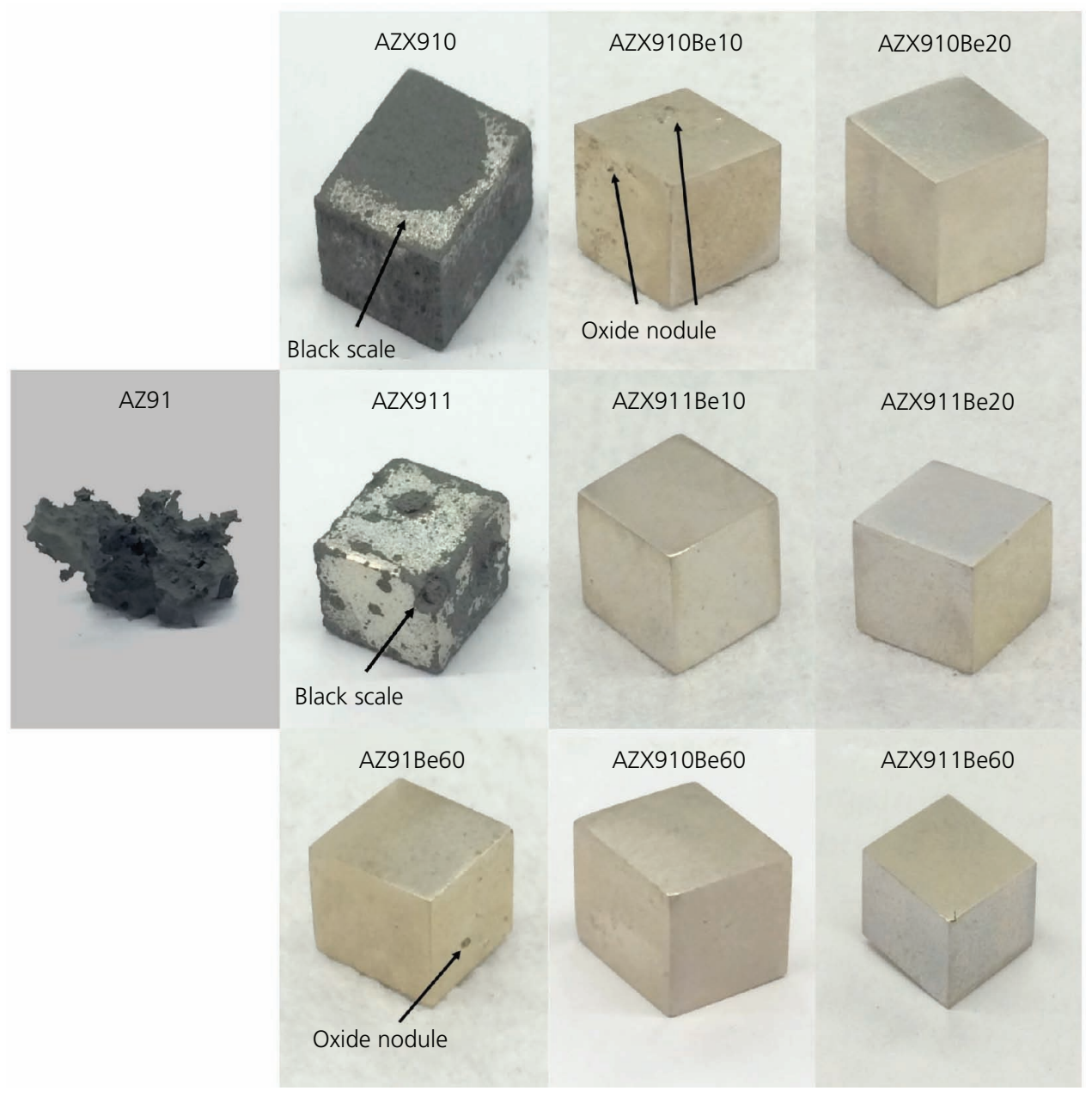

Figure 14. The macrographs of studied alloys after the LTFO test in air at $400^{\circ} \mathrm{C}$ for $144 \mathrm{~h}$. Compared with AZ91, AZX910, AZX911 and AZ91Be60 showed less oxidation. However, the oxide nodules and black scales on these alloys indicated insufficient protection of their surface oxide layers. AZX910Be20, AZX911Be10, AZX911Be20, AZX910Be60 and AZX911Be60 were completely protected, indicating the achievement of high oxidation resistance of these alloys through combined addition of beryllium and calcium. Reproduced from Tan et al. ${ }^{129}$ with permission from Elsevier

oxide-enriched magnesium oxide layer. ${ }^{129}$ This duplex layer acted as a more protective barrier to suppress both the outward diffusion of magnesium vapour and the inward penetration of oxygen compared to the reinforced magnesium oxide monolayer and the calcium oxide-enriched magnesium oxide monolayer. Based on the OR model in beryllium-containing magnesium alloys, the reinforced magnesium oxide is expected to be the beryllium-enriched $(\mathrm{Mg}, \mathrm{Be}) \mathrm{O}$ solid solution, which formed through fast outward diffusion of beryllium ions and substitution of magnesium ions with beryllium ions within magnesium oxide. ${ }^{55,69,129,148}$ The reinforced calcium oxide-( $\left.\mathrm{Mg}, \mathrm{Be}\right) \mathrm{O}$ duplex oxide layer provided higher mechanical strength/hardness to delay layer cracking during oxidation and therefore to extend effectively the oxidation incubation period. Both TEM characterisation and nanoindentation tests are needed to test this hypothesis.

\section{Concluding discussion}

Published studies indicated that the oxidation resistance of magnesium alloys is mainly governed by the incubation period, which is closely related to the crack resistance of the initially formed surface oxide layer. Layer cracking and the subsequent vigorous magnesium evaporation are major causes of catastrophic oxidation and eventual burning of magnesium alloys. Due to the incompact nature of the magnesium oxide layer $\left(R_{\mathrm{PB}}=0.81\right)$, layer cracking is easily caused by the internal tensile stresses rising during oxidation. There is a critical temperature for the non-protective oxidation of magnesium alloys, which ranges from 400 to $450^{\circ} \mathrm{C}$, depending on the alloy composition.

It has also been found that the geometrical factors, such as the surface roughness and section thickness of the sample, significantly influence the initial oxidation rate and the 
flammability of magnesium alloys. A more rapid initial oxidation rate and a higher burning tendency are caused by a higher surface roughness and a thinner section thickness of the sample, respectively. Further investigations are needed to clarify the effects of geometrical factors and therefore to standardise the testing method of the oxidation behaviours of magnesium alloys.

Microstructural modification of the magnesium oxide layer into a more protective barrier layer has long been considered as the basic concept for improving the oxidation resistance of magnesium alloys. Alloying with elements such as calcium, beryllium, strontium and REs has been confirmed as an effective method for improving the oxidation resistance of magnesium alloys. Theoretical models such as $R_{\mathrm{PB}}$, SAEE and REE have been widely used throughout the literature to rationalise the mechanism of the improved oxidation resistance. All these models only provide qualitative characterisations of the protectability of the oxide layer but cannot quantify the crack resistance of the surface oxide. In fact, high-temperature oxidation of magnesium alloys is a complex process that involves chemical reactions, ionic diffusion and phase transformation. Multiple mechanisms may work simultaneously to improve the crack resistance of the surface oxide layer. For example, the reported scattered yttriumcontaining nodules and the continuous yttrium (III) oxide layer on yttrium-containing magnesium alloys indicate that both REE and $R_{\mathrm{PB}}$ mechanisms might synergistically contribute to the improved protective capacity of the surface oxide. ${ }^{21,39,149}$

The recently proposed $\mathrm{O}_{R}$ model evidenced the correlation between the oxidation resistance of magnesium alloys with the crack resistance of their surface oxide layers, which is quantified by their mechanical strength/hardness. Experiment results confirmed that the improved oxidation resistance of beryllium-containing magnesium alloys was attributed to the formation of a fine-grained $(\mathrm{Mg}, \mathrm{Be}) \mathrm{O}$ layer with high crack resistance. This model provides a new concept in developing oxidation-resistant magnesium alloys. Techniques that can strengthen the oxide layers can improve the oxidation resistance of magnesium alloys.

Based on the current proposed models, the following criteria are formulated to select a potential alloying element $X$ to improve the oxidation resistance of magnesium alloys.

- The element $X$ should exhibit a high chemical activity.

- Surface accumulation of the element $X$ can be obtained through ionic diffusion during oxidation. The compatibility of $X$ and other solutes in the alloy should be taken into consideration to avoid the formation of $X$-containing thermostable intermetallic compounds.

- The surface oxide layer can be reinforced by the accumulated $X$ on the alloy surface by either $(a)$ forming a continuous oxide layer with high mechanical strength/hardness or (b) strengthening the initially formed magnesium oxide - for example, the formation of $(\mathrm{Mg}, X) \mathrm{O}$ solid solution.
- Surface activity, solute solubility in magnesium and the REE of the element $X$ are important factors that should also be considered during the alloy design.

At present, magnesium alloys with high ignition resistance are associated with high concentrations of calcium, strontium, yttrium, strontium, gadolinium, neodymium and dysprosium, which not only increases the alloy cost but also introduces high embrittlement susceptibilities, particularly in magnesium-aluminium-based alloys. Based on the proposed synergistic effect of beryllium and calcium, combined alloying magnesium with these alloying elements appears to be an effective method to attain the synergistic elemental effect, thereby reducing their usage in ignition-proof magnesium alloys. Further research is needed to validate their synergistic effect.

Research is also needed into the influence of alloying on the strength of the initially formed $(\mathrm{Mg}, X) \mathrm{O}$ layer.

\section{Conclusions}

- The magnesium oxide layer, with an $R_{\mathrm{PB}}$ of $0 \cdot 81$, exhibits an incompact structure with internal tension stresses. The thin magnesium oxide layer provides some protection for the substrate at relatively low temperatures. However, at temperatures above $400-450^{\circ} \mathrm{C}$, there is a non-protective oxidation stage with linear or power-law kinetics after the incubation period.

- The oxidation resistance of magnesium alloys is closely related to the protective incubation period, which is controlled by the crack resistance of the surface oxide layer. Within the incubation period, the oxidation rate is mainly governed by the ionic diffusion of magnesium ions, which is a slow process.

- The non-protective oxidation is associated with the fast, outward diffusion of magnesium vapour after surface oxide layer cracking. The nodular growth of oxide represents the end of the protective incubation period and the onset of nonprotective oxidation. Continuing oxidation or further increasing the temperature results in catastrophic oxidation and subsequent burning of magnesium alloys.

- Alloying elements (including calcium, beryllium, strontium and REs) decrease the oxidation rate and increase the $T_{\mathrm{i}}$ of magnesium alloys by forming a more protective oxide layer on the alloy surface to suppress magnesium vapourisation and oxygen penetration.

- The $R_{\mathrm{PB}}, \mathrm{SAEE}$ and REE models provide qualitative descriptions of the protective capacities of the surface oxide layer on magnesium alloys. These models provide some indications of the underlying mechanism of how the alloying elements affect the oxidation resistance of magnesium alloys.

- The OR model establishes the relation between the oxidation resistance of magnesium alloys with the mechanical strength of their surface oxide layers. A surface oxide layer with a higher mechanical strength provides a higher resistance to layer cracking during oxidation, extending the incubation period as a consequence. 
Trace addition of beryllium, even at ppm concentrations, provides significant reinforcement to the initially formed magnesium oxide layer by forming a fine-grained $(\mathrm{Mg}, \mathrm{Be}) \mathrm{O}$ solid solution. The oxide strengthening effect of beryllium effectively extends the protective incubation period of magnesium alloys.

- Combined alloying of magnesium alloys with calcium and beryllium achieves a synergistic elemental effect that significantly reduces the usage of the expensive and poisonous beryllium.

- Research is needed into the influence of alloying on the strength of the initially formed $(\mathrm{Mg}, X) \mathrm{O}$ layer.

\section{Acknowledgement}

The authors are very grateful to the University of Queensland Collaboration and Industry Engagement Fund grant for funding support (number 2014003109).

\section{REFERENCES}

1. Kwas $E$, Jürgen S, Kunert $M$ and Thein S (2017) Ceramic coatings containing calcium and phosphate on magnesium alloy MnE21. Surface Innovations 5(4): 221-231, https://doi.org/10.1680/jsuin.17.00017.

2. Jeon JJ, Lee SW, Kim BH et al. (2008) Effect of Sb and $\mathrm{Sr}$ addition on corrosion properties of $\mathrm{Mg}-5 \mathrm{Al}-2 \mathrm{Si}$ alloy. Journal of the Korean Institute of Metals and Materials 46(5): 304-309.

3. Jun JH, Kim JM, Park BK, Kim KT and Jung WJ (2005) Effects of rare earth elements on microstructure and high temperature mechanical properties of ZC63 alloy. Journal of Materials Science 40(9-10): 2659-2661.

4. Barrett CD, Imandoust A, Oppedal AL et al. (2017) Effect of grain boundaries on texture formation during dynamic recrystallization of magnesium alloys. Acta Materialia 128(15): 270-283.

5. Monteiro WA, Buso SJ and Silva LVD (2012) Application of magnesium alloys in transport. In New Features on Magnesium Alloys (Monteiro WA (ed.)). InTech, Rijeka, Croatia.

6. Lee Bray E (2018) Magnesium: Statistics and Information. US Geological Survey, Reston, VA, USA. See http://minerals.usgs.gov/ minerals/pubs/commodity/magnesium/ (accessed 24/08/2018).

7. Mordike BL and Ebert T (2001) Magnesium: properties - applications - potential. Materials Science and Engineering: A 302(1): 37-45.

8. Froes FH, Eliezer D and Aghion E (1998) The science, technology, and applications of magnesium. Journal of Metals 50(9): 30-34.

9. USAMP (US Automotive Materials Partnership) (2004) Magnesium Vision 2020: a North American Automotive Strategic Vision for Magnesium. USAMP, Southfield, MI, USA. See https://www.tms. org/Communities/FTAttachments/MG_2020___Released_11_1_[1].1. 06.pdf (accessed 24/08/2018).

10. Mathes JC (1941) Magnesium alloys in the aircraft industry. Aircraft Engineering and Aerospace Technology 13(11): 323-326.

11. Xu W, Birbilis N, Sha G et al. (2015) A high-specific-strength and corrosion-resistant magnesium alloy. Nature Materials 14(12): 1229-1235

12. Tekumalla S and Gupta M (2017) An insight into ignition factors and mechanisms of magnesium based materials: a review. Materials \& Design 113(5): 84-98.

13. Tan QY, Atrens A, Mo N and Zhang MX (2016) Oxidation of magnesium alloys at elevated temperatures in air: a review. Corrosion Science 112: 734-759.

14. Atrens A, Song GL, Liu M et al. (2015) Review of recent developments in the field of magnesium corrosion. Advanced Engineering Materials 17(4): 400-453.
15. Atrens A, Song GL, Shi Z et al. (2017) Understanding the corrosion of $\mathrm{Mg}$ and $\mathrm{Mg}$ alloys. In Reference Module in Chemistry, Molecular Sciences and Chemical Engineering (Reedijk J, Boyd R, Decker M et al. (eds)). Elsevier, Amsterdam, the Netherlands, pp. 1-20.

16. Zhang J, Zhang L, Wilke BM et al. (2017) Corrosion behaviour of microarc-oxidised magnesium alloy in Earle's balanced salt solution. Surface Innovations 5(1): 43-53, https://doi.org/10.1680/jsuin.16. 00019.

17. Bordbar-Khiabani A, Yarmand B and Mozafari M (2018) Functional PEO layers on magnesium alloys: innovative polymer-free drugeluting stents. Surface Innovations 6(4-5): 237-243, https://doi.org/ 10.1680/jsuin.18.00011.

18. Atrens A, Johnston S, Shi Z and Dargusch MS (2018) Viewpoint understanding $\mathrm{Mg}$ corrosion in the body for biodegradable medical implants. Scripta Materialia 154: 92-100.

19. Johnston S, Shi Z, Venezuela J et al. (2018) Investigating Mg biocorrosion in vitro: lessons learned and recommendations. Journal of Metals, https://doi.org/10.1007/s11837-019-03327-9.

20. Pilling NB and Bedworth RE (1923) The oxidation of metals at high temperatures. Journal of the Institute of Metals 29(4): 529-591.

21. Yu X, Jiang B, He JJ, Liu B and Pan FS (2018) Oxidation resistance of $\mathrm{Mg}-\mathrm{Y}$ alloys at elevated temperatures and the protection performance of the oxide films. Journal of Alloys and Compounds 749(15): 1054-1062.

22. Mittal M (2014) Explosion characteristics of micron- and nano-size magnesium powders. Journal of Loss Prevention in the Process Industries 27: 55-64.

23. Czerwinski $F$ (2014) Controlling the ignition and flammability of magnesium for aerospace applications. Corrosion Science 86: 1-16.

24. Boyer RR (1996) An overview on the use of titanium in the aerospace industry. Materials Science and Engineering: A 213(1): 103-114.

25. Marker T (2013) Evaluating the Flammability of Various Magnesium Alloys during Laboratory-and Full-scale Aircraft Fire Tests. Federal Aviation Administration, US Department of Transportation, Atlantic City, NJ, USA, DOT/FAA/AR-11/3.

26. Avedesian MM (ed.) (1999) Magnesium and Magnesium Alloys. ASM International, Materials Park, OH, USA.

27. Czerwinski F (ed.) (2011) Magnesium Alloys - Corrosion and Surface Treatments. InTech, Rijeka, Croatia.

28. Birks N, Meier GH and Pettit F (2006) Introduction to the High Temperature Oxidation of Metals, 2nd edn. Cambridge University Press, Cambridge, UK

29. Czerwinski $F$ (2002) The oxidation behaviour of an AZ91D magnesium alloy at high temperatures. Acta Materialia 50(10): 2639-2654.

30. López MD, Múnez CJ, Carboneras M et al. (2010) Influence of temperature on oxidation behaviour of ZE41 magnesium alloy. Journal of Alloys and Compounds 491(1-2): 131-136.

31. Lee TW, Park HW, Lim H, Kim SK and Lim SH (2017) Microstructural characterization of oxide layers in $\mathrm{CaO}$ added $\mathrm{AZ} 31 \mathrm{Mg}$ alloy. Journal of Alloys and Compounds 714: 397-408.

32. Czerwinski F (2012) Oxidation characteristics of magnesium alloys. Journal of Metals 64(12): 1477-1483.

33. Medved J, Mrvar P and Vončina M (2009) Oxidation resistance of cast magnesium alloys. Oxidation of Metals 71(5-6): 257-270.

34. Lee D, Nguyen TD and Kim YJ (2010) Oxidation of ZC63 Mg alloys reinforced with $\mathrm{SiC}$ particles between $390{ }^{\circ} \mathrm{C}$ and $500{ }^{\circ} \mathrm{C}$ in air. Metals and Materials International 16(5): 761-766.

35. Aydin DS, Baindir Z, Hoseini M and Pekguleryuz MO (2013) The high temperature oxidation and ignition behavior of $\mathrm{Mg}-\mathrm{Nd}$ alloys part I: the oxidation of dilute alloys. Journal of Alloys and Compounds 569(25): 35-44.

36. You BS, Park WW and Chung IS (2000) The effect of calcium additions on the oxidation behavior in magnesium alloys. Scripta Materialia 42(11): 1089-1094. 
37. Cheng SL, Yang GC, Fan JF, Li YJ and Zhou YH (2009) Effect of Ca and $\mathrm{Y}$ additions on oxidation behavior of AZ91 alloy at elevated temperatures. Transactions of Nonferrous Metals Society of China 19(2): 299-304.

38. Lee DB (2013) High temperature oxidation of AZ31 + 0.3 wt.\%Ca and $\mathrm{AZ31}+0.3$ wt.\%CaO magnesium alloys. Corrosion Science $\mathbf{7 0}$ 243-251.

39. Wang XM, Zeng XQ, Wu GS and Yao SS (2006) Yttrium ion implantation on the surface properties of magnesium. Applied Surface Science 253(5): 2437-2442.

40. Fan JF, Cheng SL, Xie H et al. (2005) Surface oxidation behavior of $\mathrm{Mg}-\mathrm{Y}-\mathrm{Ce}$ alloys at high temperature. Metallurgical and Materials Transactions A 36(1): 235-239.

41. Ning Z, Liang W, Cao C and Sun J (2009) The effect of $Y$ on the oxidation of $\mathrm{Mg}-\mathrm{Zn}-\mathrm{Zr}$ alloys. International Journal of Modern Physics B 23(6): 796-801.

42. Wang $X$, Wu W, Tang $Y$, Zeng $X$ and Yao S (2009) Early high temperature oxidation behaviors of $\mathrm{Mg}-10 \mathrm{Gd}-3 \mathrm{Y}$ alloys. Journal of Alloys and Compounds 474(1-2): 499-504.

43. Aydin DS, Hoseini M and Pekguleryuz MO (2014) Understanding the high temperature oxidation and ignition behaviour of two-phase $\mathrm{Mg}-\mathrm{Nd}$ alloys and a comparison to single phase $\mathrm{Mg}-\mathrm{Nd}$. Philosophical Magazine 95(3): 259-274.

44. Zhao S, Zong Z, Zhou T et al. (2013) The oxidation resistance and ignition temperature of AZ31 magnesium alloy with additions of $\mathrm{La}_{2} \mathrm{O}_{3}$ and La. Corrosion Science 67: 75-81.

45. Tan QY, Mo N, Jiang B et al. (2016) Oxidation resistance of Mg-9Al-1Zn alloys micro-alloyed with Be. Scripta Materialia 115(1): $38-41$

46. Aydin DS, Bayindir Z and Pekguleryuz MO (2015) High temperature oxidation behavior of hypoeutectic $\mathrm{Mg}-\mathrm{Sr}$ binary alloys: the role of the two-phase microstructure and the surface activity of Sr. Advanced Engineering Materials 17(5): 697-708.

47. Nguyen TD, Kim YJ, Lee JC, Kim SJ and Lee DB (2010) Effect of dispersed $\mathrm{SiC}$ particles on the oxidation of AZ91D magnesium alloys between 420 and $500^{\circ} \mathrm{C}$ in air. Surface Review and Letters 17(10): 9-14.

48. Lin PY, Zhou H, Li WP, Zhao SZ and Su JG (2009) Effect of yttrium addition on the oxide scale of AM50 magnesium alloy. Corrosion Science 51(5): 1128-1133.

49. Choi BH, You BS, Park WW, Huang YB and Park LM (2003) Effect of $\mathrm{Ca}$ addition on the oxidation resistance of AZ91 magnesium alloys at elevated temperatures. Metals and Materials International 9(4): 395-398.

50. Nguyen T and Lee D (2010) Oxidation of AM60B Mg alloys containing dispersed $\mathrm{SiC}$ particles in air at temperatures between 400 and $550^{\circ} \mathrm{C}$. Oxidation of Metals 73(1-2): 183-192.

51. $\mathrm{Yu} \mathrm{X}$, Jiang $\mathrm{B}$, Yang $\mathrm{H}$ et al. (2015) High temperature oxidation behavior of $\mathrm{Mg}-\mathrm{Y}-\mathrm{Sn}, \mathrm{Mg}-\mathrm{Y}, \mathrm{Mg}-\mathrm{Sn}$ alloys and its effect on corrosion property. Applied Surface Science 353(30): 1013-1022.

52. Lee DB (2016) Effect of $\mathrm{CaO}$ and hot extrusion on the oxidation of AZ61 magnesium alloys. Oxidation of Metals 85(1): 65-74.

53. Jang DI and Kim SK (2016) Effect of $\mathrm{Ca}(\mathrm{OH})_{2}$ on oxidation and ignition resistances of pure $\mathrm{Mg}$. In Essential Readings in Magnesium Technology (Mathaudhu SN, Luo AA, Neelameggham NR, Nyberg and Sillekens WH (eds)). Springer, Cham, Switzerland, pp. 145-149.

54. Barrena MI, Gómez de Salazar JM, Matesanz L and Soria A (2011) Effect of heat treatment on oxidation kinetics in AZ91 and AM60 magnesium alloys. Materials Characterization 62(10): 982-986

55. Tan QY, Mo N, Lin CL et al. (2019) Generalisation of the oxide reinforcement model for the high oxidation resistance of some $\mathrm{Mg}$ alloys micro-alloyed with Be. Corrosion Science 147: 357-371.
56. Toda-Caraballo I, Galindo-Nava El and Rivera-Diaz-del-Castillo PEJ (2014) Understanding the factors influencing yield strength on $\mathrm{Mg}$ alloys. Acta Materialia 75(15): 287-296.

57. Lide DR (2003) Properties of the elements and inorganic compounds; vapor pressure of the metallic elements. CRC Handbook of Chemistry and Physics (Lide DR (ed.)). 84th edn. CRC Press, Boca Raton, FL, USA.

58. Arrabal R, Pardo A, Merino MC et al. (2011) Oxidation behavior of AZ91D magnesium alloy containing $\mathrm{Nd}$ or Gd. Oxidation of Metals 76(5-6): 433-450.

59. Ning ZL, Zhao XY, Luo AA et al. (2017) The melt protection mechanism of an $\mathrm{SO}_{2} / \mathrm{CO}_{2}$ gas mixture for a magnesium-rare-earth based alloy. Journal of Alloys and Compounds 722: 101-107.

60. Czerwinski F (2015) The reactive element effect on high-temperature oxidation of magnesium. International Materials Reviews 60(5): 264-296.

61. Przeliorz R and Piatkowski (2012) On the oxidation behaviour of WE43 and MSR-B magnesium alloys in $\mathrm{CO}_{2}$ atmosphere. Solid State Phenomena 191: 154-161.

62. Czerwinski F (2004) Factors affecting the oxidation nature of magnesium alloys. Journal of Metals 56(5): 29-31.

63. Zhang ZJ, Fu, XQ, Mao MM et al. (2016) In situ observation of sublimation-enhanced magnesium oxidation at elevated temperature. Nano Research 9(9): 2796-2802.

64. Eubanks AG, Moore DG and Pennington WA (1962) Effect of surface roughness on the oxidation rate of iron. Journal of the Electrochemical Society 109(5): 382-389.

65. Wang Li, Jiang WG, Li XW et al. (2015) Effect of surface roughness on the oxidation behavior of a directionally solidified Ni-based superalloy at $1,100^{\circ} \mathrm{C}$. Acta Metallurgica Sinica (English Letters) 28(3): 381-385.

66. Li Z, Qian S and Wang W (2011) Influence of superalloy substrate roughness on adhesion and oxidation behavior of magnetron-sputtered NiCoCrAlY coatings. Applied Surface Science 257(24): 10414-10420.

67. Huntz AM, Lefevre B and Cassino F (2000) Roughness and oxidation: application to $\mathrm{NiO}$ growth on $\mathrm{Ni}$ at $800^{\circ} \mathrm{C}$. Materials Science and Engineering: A 290(1): 190-197.

68. Walter R and Kannan MB (2011) Influence of surface roughness on the corrosion behaviour of magnesium alloy. Materials \& Design 32(4): 2350-2354.

69. Tan QY, Mo N, Lin CL et al. (2018) Improved oxidation resistance of $\mathrm{Mg}-9 \mathrm{Al}-1 \mathrm{Zn}$ alloy microalloyed with $60 \mathrm{wt}$ ppm Be attributed to the formation of a more protective $(\mathrm{Mg}, \mathrm{Be}) \mathrm{O}$ surface oxide. Corrosion Science 132: 272-283.

70. White EL and Ward JJ (1966) Ignition of Metals in Oxygen. Defense Metals Information Center, Columbus, OH, USA.

71. Tekumalla S, Nandigam Y, Bibhanshu N et al. (2018) A strong and deformable in-situ magnesium nanocomposite igniting above $1000^{\circ}$ C. Scientific Reports 8(1): 1-10.

72. Arrabal R, Pardo A, Mohedano M et al. (2012) Oxidation behaviour of AM50 magnesium alloy containing lanthanide lements. Materials and Corrosion 65(6): 582-592.

73. Li YY, Zhao WM, Xue HT and Jian D (2012) Study on Magnesium Alloys Ignition Temperature Test System. Springer, Berlin, Germany.

74. Czerwinski F (2014) Overcoming barriers of magnesium ignition and flammability. Advanced Materials \& Processes 172(5): 28-31.

75. Kim S (2011) Design and development of high-performance Eco-Mg alloys. In Magnesium Alloys: Design, Processing and Properties (Czerwinski F (ed.)). InTech, Rijeka, Croatia.

76. Liu M, Donald SS, Parish C and Atrens A (2012) The ignition temperature of $\mathrm{Mg}$ alloys WE43, AZ31 and AZ91. Corrosion Science 54: 139-142.

77. Lin P, Zhou H, Li W et al. (2008) Interactive effect of cerium and aluminum on the ignition point and the oxidation resistance of magnesium alloy. Corrosion Science 50(9): 2669-2675. 
78. Fan JF, Chen ZY, Yang WD, Fang S and Xu BR (2012) Effect of yttrium, calcium and zirconium on ignition-proof principle and mechanical properties of magnesium alloys. Journal of Rare Earths 30(1): 74-78.

79. Bobryshev BL and Aleksandrova YP (1988) Ignition of magnesium and its alloys. Metal Science and Heat Treatment 30(3): 219-222.

80. Fassell WM, Gulbransen LB, Lewis JR and Hamilton JH (1951) Ignition temperatures of magnesium and magnesium alloys. Journal of Metals 3(7): 522-528.

81. Zhou N, Zhang ZY, Dong J, Jin L and Ding WJ (2013) Selective oxidation behavior of an ignition-proof $\mathrm{Mg}-\mathrm{Y}-\mathrm{Ca}-\mathrm{Ce}$ alloy. Journal of Rare Earths 31(10): 1003-1008.

82. Fan JF, Yang CL, Han G et al. (2011) Oxidation behavior of ignitionproof magnesium alloys with rare earth addition. Journal of Alloys and Compounds 509(5): 2137-2142.

83. Huang YB, Chung IS, You BS, Park WW and Choi BH (2004) Effect of $\mathrm{Be}$ addition on the oxidation behavior of $\mathrm{Mg}-\mathrm{Ca}$ alloys at elevated temperature. Metals and Materials International 10(1): $7-11$.

84. Aydin DS, Bayindir A and Pekguleryuz MO (2013) The effect of strontium (Sr) on the ignition temperature of magnesium $(\mathrm{Mg})$ : a look at the pre-ignition stage of $\mathrm{Mg}-6 \mathrm{wt} \% \mathrm{Sr}$. Journal of Materials Science 48(23): 8117-8132.

85. Tekumalla S, Chen Y, Seetharaman S et al. (2016) Enhancing overall static/dynamic/damping/ignition response of magnesium through the addition of lower amounts $(<2 \%)$ of yttrium. Journal of Alloys and Compounds 689(36): 350-358.

86. Zhao WM, Yong S, Li H and Liang C (2006) The effects of some elements on the igniting temperature of magnesium alloys. Materials Science and Engineering: B 127(2-3): 105-107.

87. Inoue S, Yamasaki M and Kawamura Y (2017) Formation of an incombustible oxide film on a molten $\mathrm{Mg}-\mathrm{Al}-\mathrm{Ca}$ alloy. Corrosion Science 122(1): 118-122.

88. Tan XH, How W, Weng J, Onn R and Gupta M (2015) Development of high-performance quaternary LPSO $\mathrm{Mg}-\mathrm{Y}-\mathrm{Zn}-\mathrm{Al}$ alloys by disintegrated melt deposition technique. Materials \& Design 83 : 443-450.

89. Liu CC, Lu S, Fu YT and Zhang HP (2015) Flammability and the oxidation kinetics of the magnesium alloys AZ31, WE43, and ZE10. Corrosion Science 100: 177-185.

90. Prasad A, Shi ZM and Atrens A (2012) Influence of Al and $Y$ on the ignition and flammability of $\mathrm{Mg}$ alloys. Corrosion Science $\mathbf{5 5}$ : 153-163.

91. Mebarki N, Ravi Kumar NV, Blandin JJ et al. (2005) Correlation between ignition and oxidation behaviours of AZ91 magnesium alloy. Materials Science and Technology 21(10): 1145-1151.

92. Li F, Peh WY, Nagarajan $V$ et al. (2016) Development of nonflammable high strength AZ91+Ca alloys via liquid forging and extrusion. Materials \& Design 99: 37-43.

93. Han YF, Liu T and Zhang Q (2014) Effect of Nd and Dy addition on AZ91D ignition temperature and microhardness. Materials Science Forum 788: $34-40$.

94. Lee JK and Kim SK (2011) Effect of $\mathrm{CaO}$ addition on the ignition resistance of Mg-Al alloys. Materials Transactions 52(7): 1483-1488.

95. Kasprzak W, Sokolowski JH, Sahoo M and Dobrzanski LA (2008) Thermal and structural characteristics of the AM50 magnesium alloy. Journal of Achievements in Materials and Manufacturing Engineering 28(2): 131-138.

96. Kumar NVR, Blandin JJ, Suery M and Grosjean E (2003) Effect of alloying elements on the ignition resistance of magnesium alloys. Scripta Materialia 49(3): 225-230.

97. Czerwinski $F$ (2015) Role of surface reactivity in processing and performance of magnesium alloys. Materials Science Forum 828-829: 401-406.
98. Choi BH, You BS and Park IM (2006) Characterization of protective oxide layers formed on molten AZ91 alloy containing Ca and Be. Metals and Materials International 12(1): 63-67.

99. Fan JF, Yang GC, Zhou YH, Wei YH and Xu BS (2009) Selective oxidation and the third-element effect on the oxidation of $\mathrm{Mg}-\mathrm{Y}$ alloys at high temperatures. Metallurgical and Materials Transactions A 40(9): 2184-2189.

100. Wu YJ, Peng LM, Zhao A et al. (2012) Ignition-proof properties of a high-strength $\mathrm{Mg}-\mathrm{Gd}-\mathrm{Ag}-\mathrm{Zr}$ alloy. Journal of Shanghai Jiaotong University (Science) 17(6): 643-647.

101. Cheng C, Lan Q, Wang A et al. (2018) Effect of Ca additions on ignition temperature and multi-stage oxidation behavior of AZ80. Metals 8(10): 766-777.

102. Polmear IJ (ed.) (1995) Light Alloys, 3rd edn. Arnold, London, UK.

103. Okamoto H (ed.) (2000) Phase Diagrams for Binary Alloys. ASM International, Materials Park, OH, USA.

104. Zhang MX and Kelly PM (2003) Crystallography of Mg17A112 precipitates in AZ91D alloy. Scripta Materialia 48(5): 647-652.

105. Zeng G, Xian JW and Gourlay CM (2018) Nucleation and growth crystallography of A18Mn5 on B2-Al(Mn,Fe) in AZ91 magnesium alloys. Acta Materialia 153: 364-376.

106. Luo A and Pekguleryuz MO (1994) Cast magnesium alloys for elevated temperature applications. Journal of Materials Science 29(20): 5259-5271.

107. Cheng S, Yang G, Fan J, Li Y and Zhou Y (2006) Effect of Ca and Y on microstructure and mechanical properties of AZ91 alloy. Rare Metal Materials and Engineering 35(9): 1400-1403.

108. Shih TS, Wang JH and Chong KZ (2004) Combustion of magnesium alloys in air. Materials Chemistry and Physics 85(2-3): 302-309.

109. Li P, Tang B and Kandalova EG (2005) Microstructure and properties of AZ91D alloy with Ca additions. Materials Letters 59(6): 671-675.

110. Marker T (2014) Development of a Laboratory-scale Flammability Test for Magnesium Alloys Used in Aircraft Seat Construction. Federal Aviation Administration, US Department of Transportation, Atlantic City, NJ, USA, DOT/FAA/TC-13/52

111. Prasad A, Shi ZM and Atrens A (2012) Flammability of $\mathrm{Mg}-\mathrm{X}$ binary alloys. Advanced Engineering Materials 14(9): 772-784.

112. Yu Q, Mao MM, Li QJ et al. (2016) In situ observation on dislocation-controlled sublimation of $\mathrm{Mg}$ nanoparticles. Nano Letters 16(2): 1156-1160.

113. Kim YM, Yim CD, Kim HS and You BS (2011) Key factor influencing the ignition resistance of magnesium alloys at elevated temperatures. Scripta Materialia 65(11): 958-961.

114. Zeng XQ, Want QD, Lu YZ et al. (2001) Behavior of surface oxidation on molten $\mathrm{Mg}-9 \mathrm{Al}-0.5 \mathrm{Zn}-0.3 \mathrm{Be}$ alloy. Materials Science and Engineering: A 301(2): 154-161.

115. Wang XM, Zeng XQ, Wu GS, Yao SS and Lai YJ (2008) The effects of cerium implantation on the oxidation behavior of AZ31 magnesium alloys. Journal of Alloys and Compounds 456(1): 384-389.

116. Chen $Z$, Ren $X$ and Zhang $Y$ (2005) Effect of RE on the ignitionproof, microstructure and properties of AZ91D magnesium alloy. Journal of University of Science and Technology Beijing: Mineral Metallurgy Materials (English Edition) 12(6): 540-544.

117. Fan JF, Yang GX, Chen SL et al. (2004) Effect of rare earths (Y, Ce) additions on the ignition points of magnesium alloys. Journal of Materials Science 39(20): 6375-6377.

118. Sakamoto M, Akiyama S and Oqi K (1997) Suppression of ignition and burning of molten $\mathrm{Mg}$ alloys by $\mathrm{Ca}$ bearing stable oxide film. Journal of Materials Science Letters 16(12): 1048-1050.

119. Houska C (1988) Beryllium in aluminium and magnesium alloys. Metals and Materials 4(2): 100-104.

120. Czerwinski $F$ (2004) The early stage oxidation and evaporation of Mg-9\%Al-1\%Zn alloy. Corrosion Science 46(2): 377-386. 
121. Zeng $X Q$, Wand QD, Lü $Y Z$ et al. (2000) Study on ignition proof magnesium alloy with beryllium and rare earth additions. Scripta Materialia 43(5): 403-409.

122. Kiejna A and Wojciechowski KF (1983) Surface properties of alkalimetal alloys. Journal of Physics C: Solid State Physics 16(35): 6883-6896.

123. Kiejna A (1990) Comment on the surface segregation in alkali-metal alloys. Journal of Physics: Condensed Matter 2(29): 6331-6333.

124. Yamauchi H (1985) Surface segregation in jellium binary solid solutions. Physical Review B 31(12): 7688-7694.

125. Ren FZ, Cao K, Ren JZ et al. (2014) Numerical calculation of the electron density at the Wigner-Seitz radius based on the Thomas-Fermi-Dirac equation. Journal of Computational and Theoretical Nanoscience 11(2): 1-4.

126. Pekguleryuz M and Vermette P (2004) Strontium for Melt oxidation Reduction of Magnesium and a Method for Adding Strontium to Magnesium. US Patent US20040159188 A1, Aug.

127. Pekguleryuz M and Avedesian M (1992) Magnesium alloying some metallurgical aspects. In Magnesium Alloys and Their Applications (Kainer KU (ed.)). DGM, Garmisch, Germany, pp. 213-220.

128. Sakamoto M, Akiyama S, Hagio T and Ogi K (1997) Control of oxidation surface film and suppression of ignition of molten $\mathrm{Mg}-\mathrm{Ca}$ alloy by Ca addition. Journal of Japan Foundry Engineering Society 69(3): 227-233.

129. Tan QY, Mo N, Jiang B et al. (2017) Combined influence of $\mathrm{Be}$ and $\mathrm{Ca}$ on improving the high-temperature oxidation resistance of the magnesium alloy Mg-9Al-1Zn. Corrosion Science 122(1): $1-11$.

130. Pfeil L (1937) Improvement of Heat-resisting Alloys, UK Patent 459 848, May.

131. Roure S, Czerwinski $F$ and Petric A (1994) Influence of $\mathrm{CeO}_{2}$-coating on the high-temperature oxidation of chromium. Oxidation of Metals 42(1): $75-102$

132. Stringer J (1989) The reactive element effect in high-temperature corrosion. Materials Science and Engineering: A 120-121: 129-137.

133. He J, Peng H, Gong SK and Guo HB (2017) Synergistic effect of reactive element co-doping in two-phase $\left(\gamma^{\prime}+\beta\right) \mathrm{Ni}-\mathrm{Al}$ alloys. Corrosion Science 120: 130-138.

134. Czerwinski F and Szpunar JA (1998) The influence of crystallographic orientation of nickel surface on oxidation inhibition by ceria coatings. Acta Materialia 46(4): 1403-1417.
135. Czerwinski $F$ (2000) On the use of the micromarker technique for studying the growth mechanism of thin oxide films. Acta Materialia 48(3): 721-733.

136. Kofstad P (ed.) (1988) High Temperature Corrosion. Elsevier, New York, NY, USA

137. Wells AF (ed.) (1984) Structural Inorganic Chemistry, 5th edn. Clarendon Press, Oxford, UK.

138. Jung YG, Lawn BR, Martyniuk M, Huang H and Hu XZ (2004) Evaluation of elastic modulus and hardness of thin films by nanoindentation. Journal of Materials Research 19(10): 3076-3080.

139. Campbell FC (2008) Elements of Metallurgy and Engineering Alloys. ASM International, Materials Park, OH, USA.

140. Misra A, Sharif AA, Petrovic JJ and Mitchell TE (2000) Rapid solution hardening at elevated temperatures by substitutional Re alloying in $\mathrm{MoSi}_{2}$. Acta Materialia 48(4): 925-932.

141. Zhou YC, Chen JX and Wang JY (2006) Strengthening of $\mathrm{Ti}_{3} \mathrm{AlC}_{2}$ by incorporation of $\mathrm{Si}$ to form $\mathrm{Ti}_{3} \mathrm{Al}_{1-x} \mathrm{Si}_{x} \mathrm{C}_{2}$ solid solutions. Acta Materialia 54(5): 1317-1322.

142. Ali Y, Qiu D, Jiang B, Pan FS and Zhang MX (2015) Current research progress in grain refinement of cast magnesium alloys: a review article. Journal of Alloys and Compounds 619: 639-651.

143. Krell A and Blank P (1996) The influence of shaping method on the grain size dependence of strength in dense submicrometre alumina. Journal of the European Ceramic Society 16(11): 1189-1200.

144. Brook GB (1998) General physical properties of light metal alloys and pure light metals. In Smithells Light Metals Handbook (Smithells CJ and Brook GB (eds)). Butterworth-Heinemann, Oxford, UK, pp. 5-13.

145. Yu XW, Shen SJ et al. (2016) The effect of the existing state of Y on high temperature oxidation properties of magnesium alloys. Applied Surface Science 370: 357-363.

146. Haley PJ, Finch GL, Hoover MD and Cuddihy RG (1990) The acute toxicity of inhaled beryllium metal in rats. Fundamental and Applied Toxicology 15(4): 767-778.

147. Cooper RG and Harrison AP (2009) The uses and adverse effects of beryllium on health. Indian Journal of Occupational and Environmental Medicine 13(2): 65-76.

148. Van Orman JA and Crispin KL (2010) Diffusion in oxides. Reviews in Mineralogy and Geochemistry 72(1): 757-825.

149. Yu X, Jiang B, He JJ et al. (2016) Effect of $\mathrm{Zn}$ addition on the oxidation property of $\mathrm{Mg}-\mathrm{Y}$ alloy at high temperatures. Journal of Alloys and Compounds 687: 252-262.

\section{How can you contribute?}

To discuss this paper, please submit up to 500 words to the journal office at journals@ice.org.uk. Your contribution will be forwarded to the author(s) for a reply and, if considered appropriate by the editor-in-chief, it will be published as a discussion in a future issue of the journal.

ICE Science journals rely entirely on contributions from the field of materials science and engineering. Information about how to submit your paper online is available at www.icevirtuallibrary.com/page/authors, where you will also find detailed author guidelines. 